250538
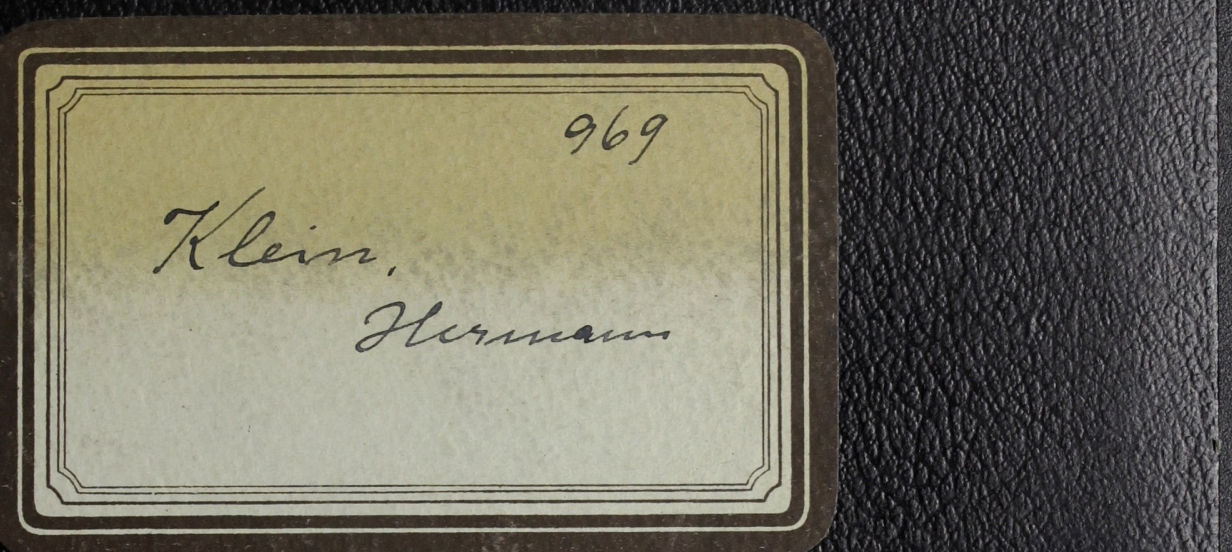

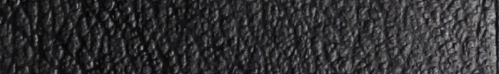

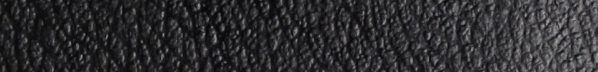

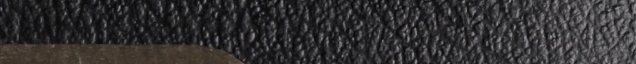

969 (1) 30 .

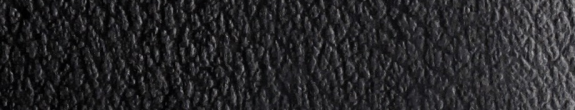
1) 17.

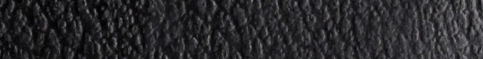
Who Hix $1, \ldots$ 6. (ans) (8. for (x) 601. 17.

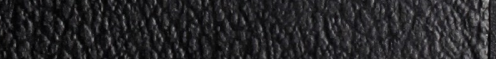
Wun 2. . W

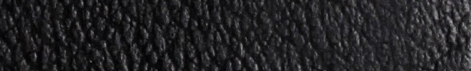

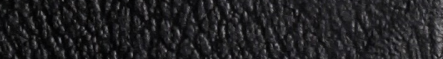
(3) 


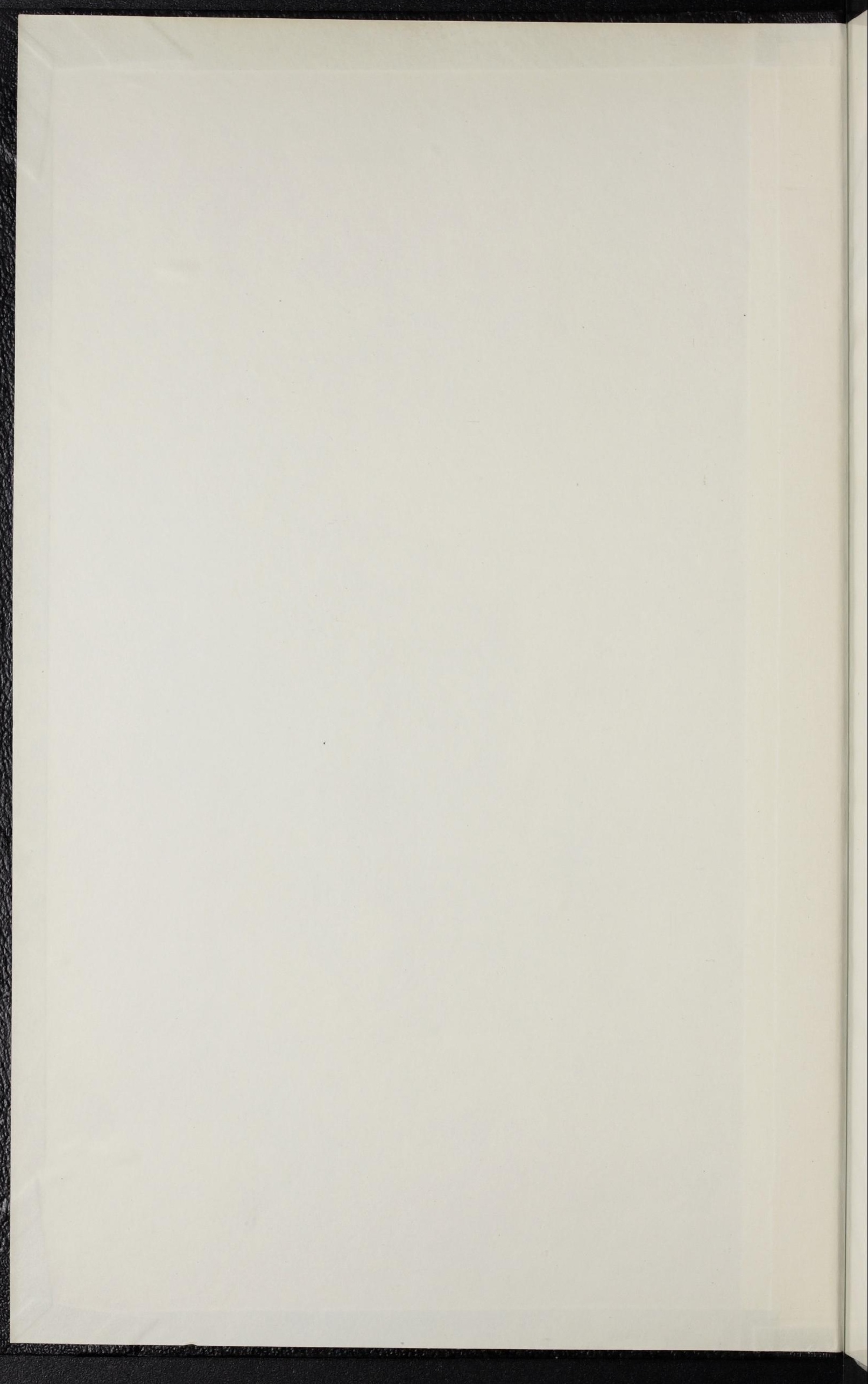





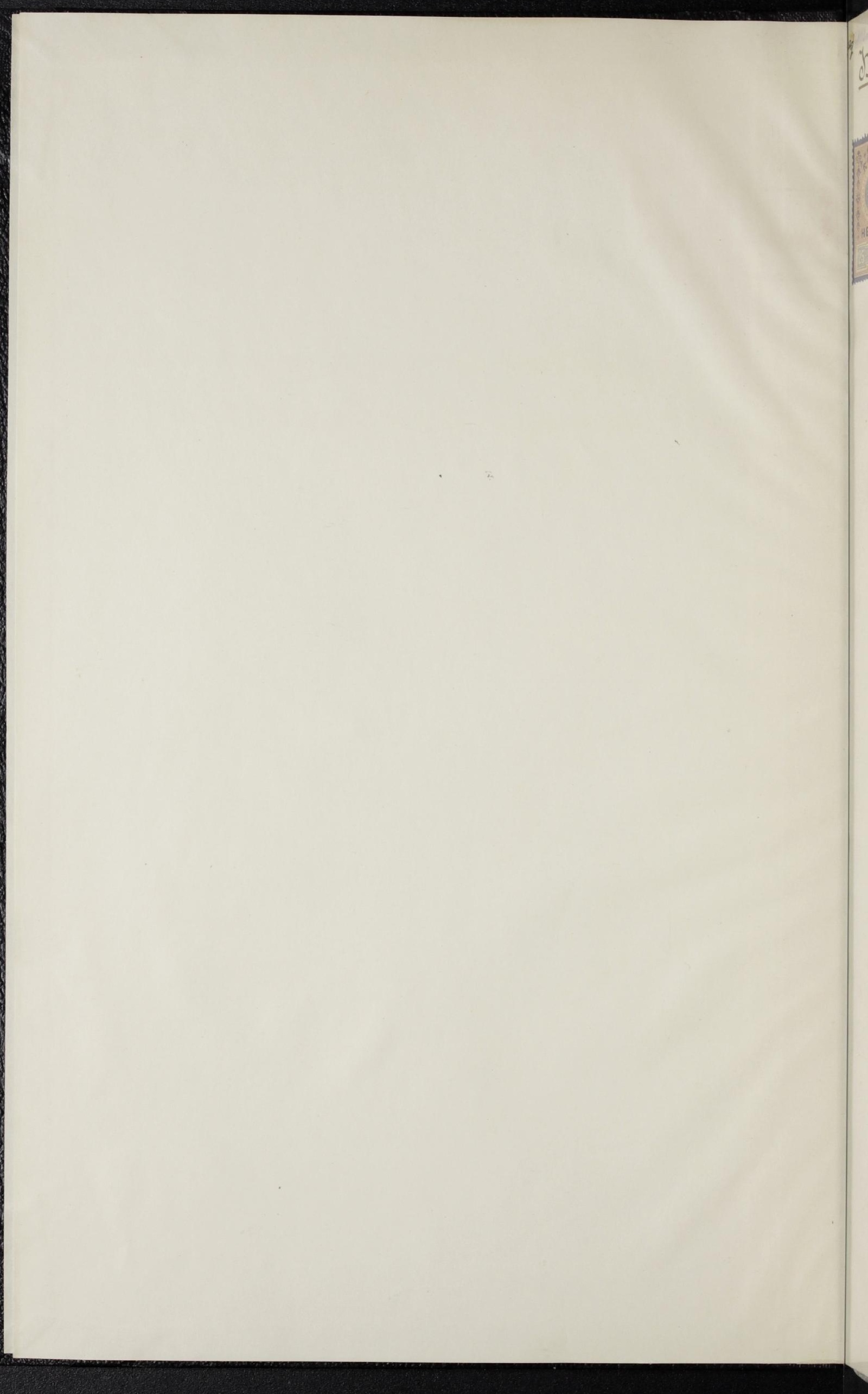




\section{Jermann Klecin}

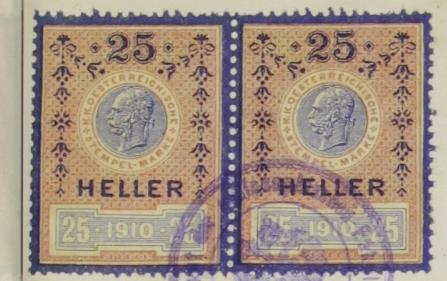

Prig. Sorick: YOOP.

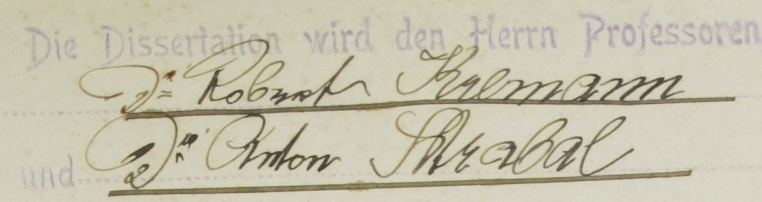

Graz, an 22 forminan 191\%. Martinar

1. Eur Syntbese der naturlicben Jette vom Standpunkt der Pbosentebre. 


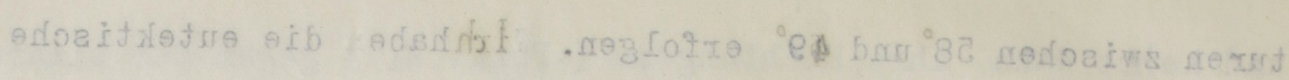

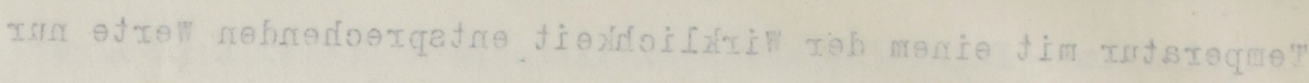

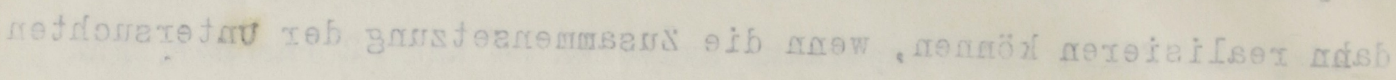

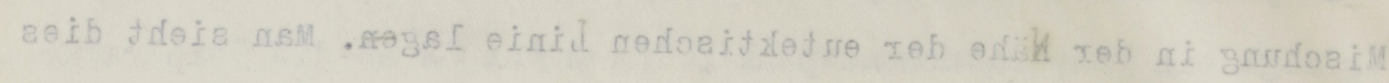

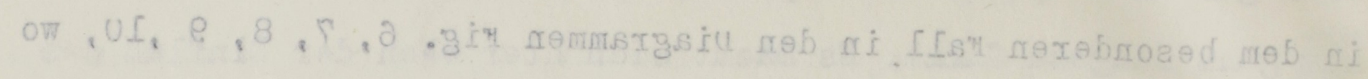
=usfoenstov revers

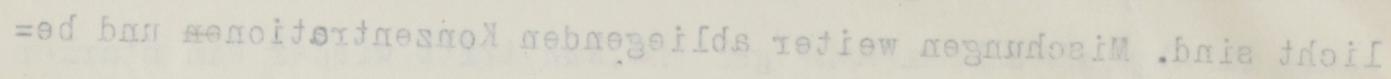

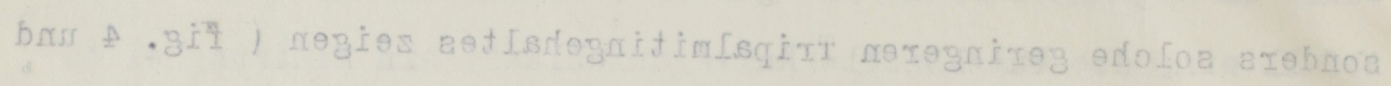

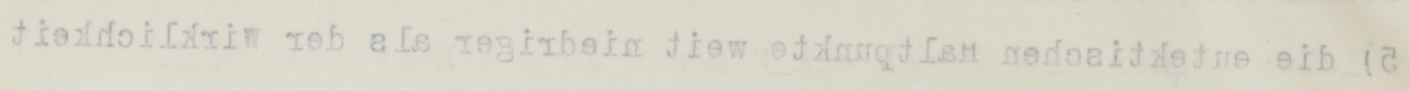

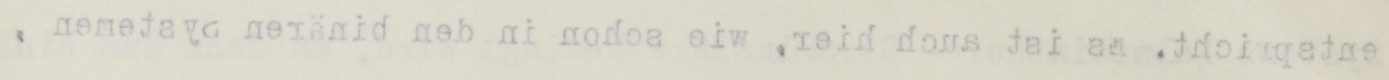

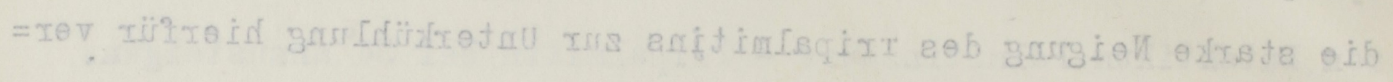

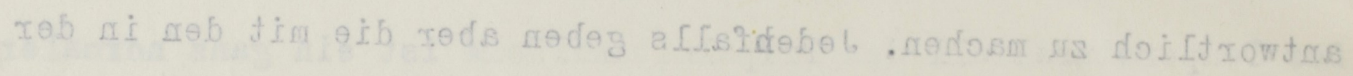

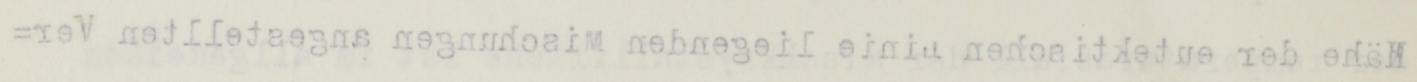

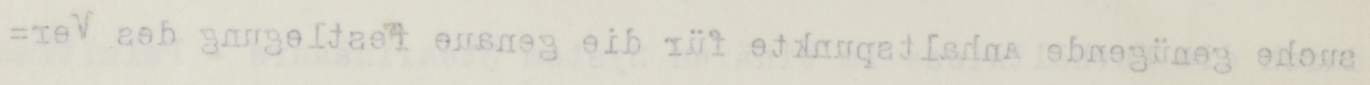

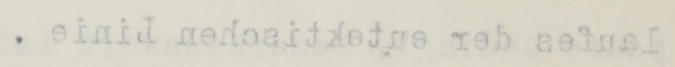


Sermann Hilein

ad Rigivetsel=969

adfores 461 ex1916) 18

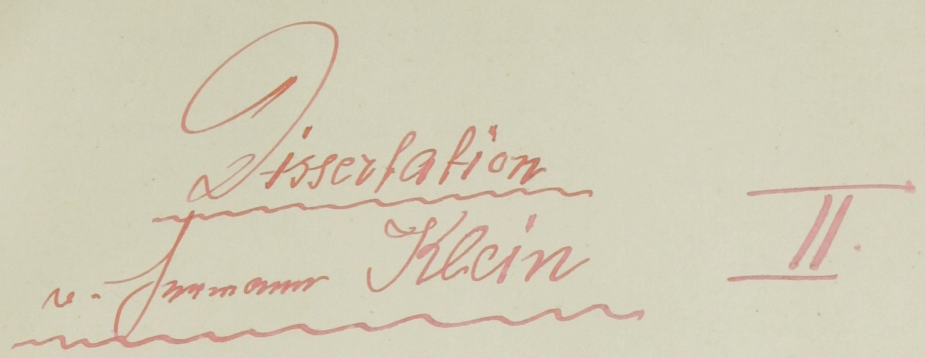

2. Zur dinctik der Furfurolbildung ons Bentosen (Orabinose). 


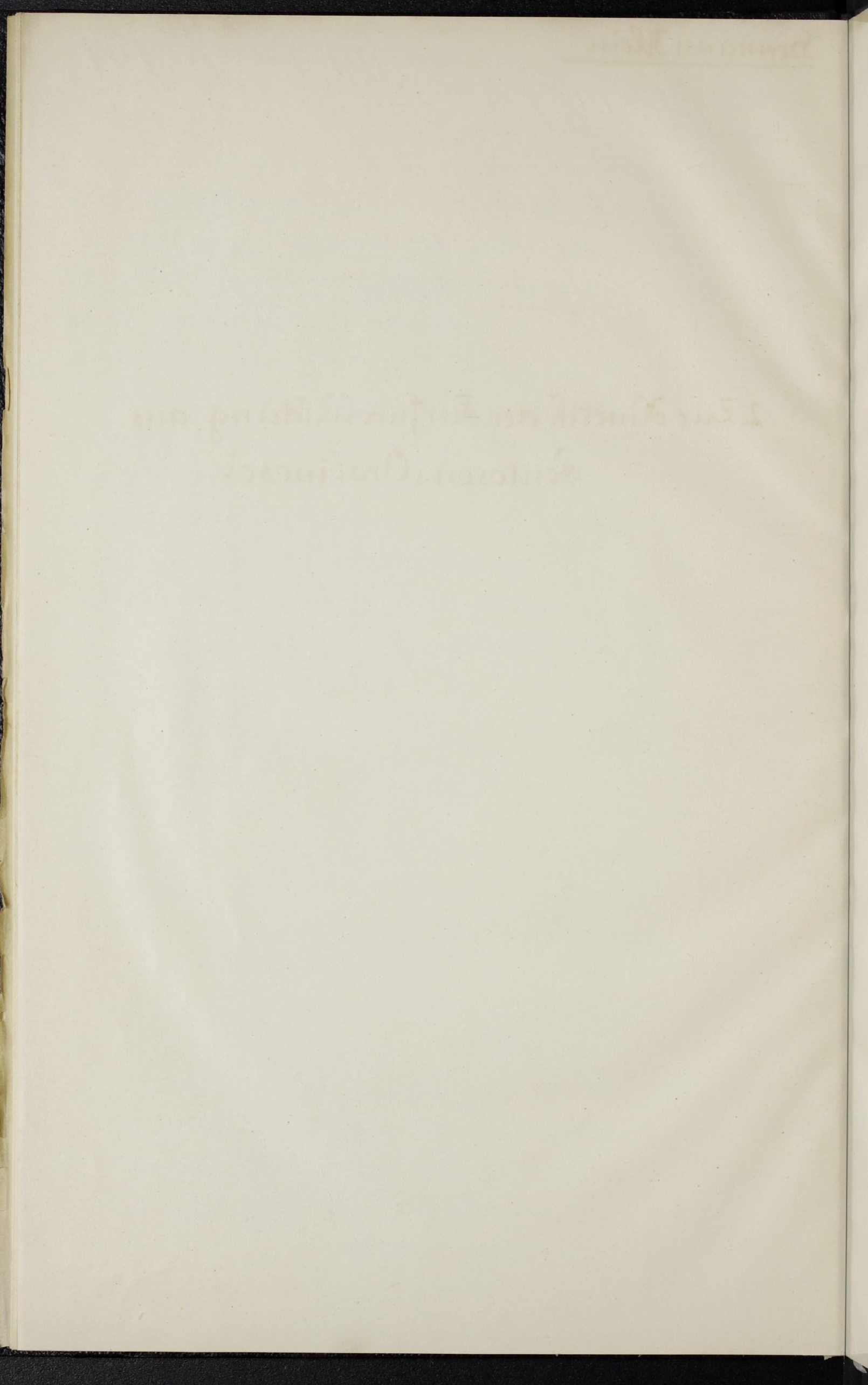


Zur Kinetik der Furfurolbildung aus

\author{
Pentosen (Arabinose) \\ Von \\ He rma $n$ n $\mathbb{R} 1$ e i \\ ( mit 2 Textfiguren)
}

Aus dem Chemischen Institut der Universität Graz.

Bekanntlich beruht die quantitative Bestimmung von Pentosen auf der Tatsache, dass diese bei der Destillation mit starker Salz oder Schwefelsäure quantitativ in Furfurol übergefüht werden, dessen Menge im Destillat durch Fällung mit Fhenylhydrazin, Pyrogallol, Phloroglucin, Barbitursäure oder am zweckmässigsten mit Semioxamazid bestimmt werden kann. Betrachten wir das Reaktionsschema der Furfurolbildung aus Pentosen:

$$
\mathrm{C}_{5} \mathrm{H}_{10} \mathrm{O}_{5} \rightarrow \mathrm{C}_{5} \mathrm{H}_{4} \mathrm{O}_{2}+3 \mathrm{H}_{2} \mathrm{O} \text {, }
$$

so sehen wir, dass es sich um eine reine Wasserabspaltung handelt, ähnlich wie es z.B. bei der Esterbildung aus organischen Sëuren mit einem grossen Alkoholüberschuss der Fall ist. Die Säure selbst nimmt an der Reaktion scheinbar nicht teil.

Es lag nun der Gedanke nahe, dass der Vorgang der Furfurol bildung in $w$ ä s s e r i g e $r$ Lösung, ähnlich wie der der Esterbildung unter den oben erwähnten Bedingungen, ein freiwillig und praktisch vollständig verlaufender Vorgang ist, der bei entsprechend hoher Temperatur und infolge gleichzeitiger katalytischer Beschleunigung durch Wasserstoffionen mit einer messbaren Geschwindigkeit verläuft, die ausreichend ist, dass die Furfurolbildung aus Fentosen zu einer quantitativen Bestimmungsmethode Verwendung finden kann. Zwecks Prüfung der einschlägigen Verhältnisse habe ich die 


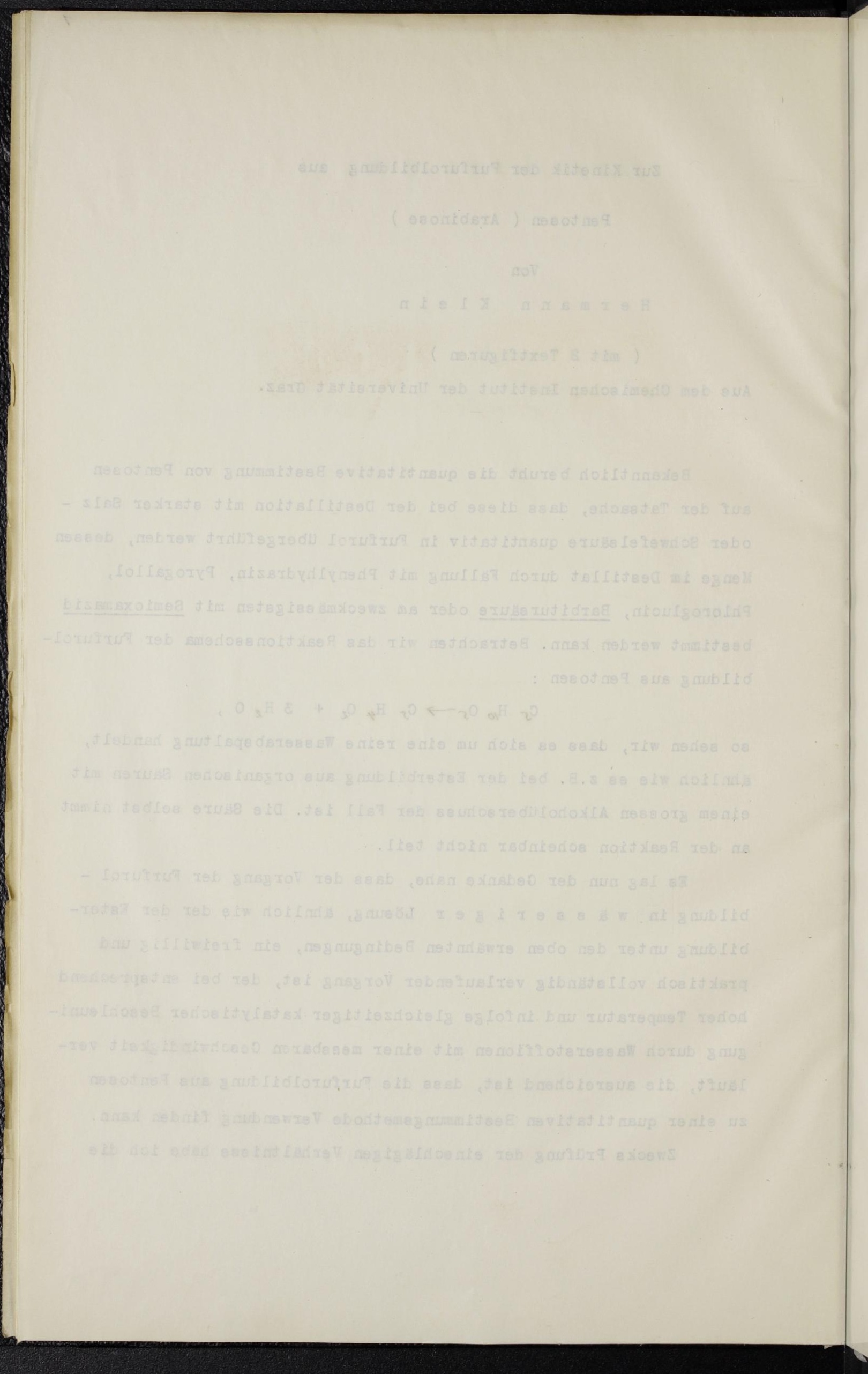


Furfurolbildung aus einer 0.35 molaren Arabinoselösung bei $95^{\circ}$ in geschlossen en Gefässen in 3.13, 1.57 und 0.313 norm. $\mathrm{H}_{2} \mathrm{SO}_{4}$, beziehungsweise 2.90 norm. Salz säure zeitlich verfolgt.

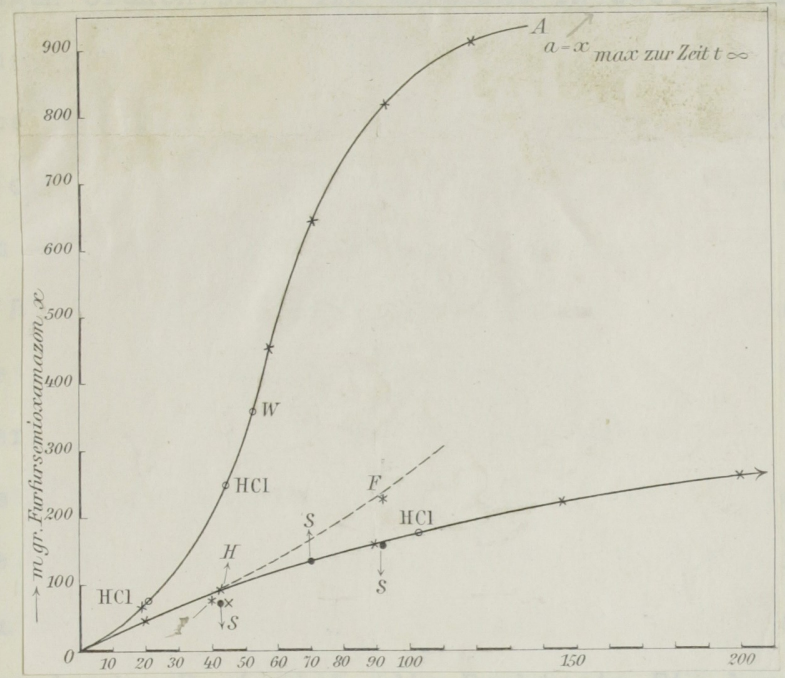

Fig. 1

Die bei den Versuchen mit 3.13 norm. $\mathrm{H}_{2} \mathrm{SO}_{4}$, beziehungsweise 2.9 norm. HCI - solche Säurekonzentrationen entsprechen ungefähr den bei der quantitativen Pentosebestimmung verwendeten Säurekonzentrationen ( zirka 12 prozentige HCI ) - zu verschiedenen Zeiten entstandenen Mengen Furfurol, ausgedrückt in Milligramm Furfursemioxamazon, sind in Fig. I in Abhängigkeit von der Reaktionsdauer graphisch dar gestellt.

Die Versuche mit 3.13 norm. $\mathrm{H}_{2} \mathrm{SO}_{4}$ sind in der Fig. 1 mit $x$ eingetragen (Versuche der Tabelle I). Fur die weitere Deutung der Versuchsergebnisse ist die Bemerkung nötig, dass die Glasphiolen, in welche die stets releutische Lösung des Reaktionsgemisches einge schlossen wurde, teils neu, teils zu meinen Versuchen bereits ver- 


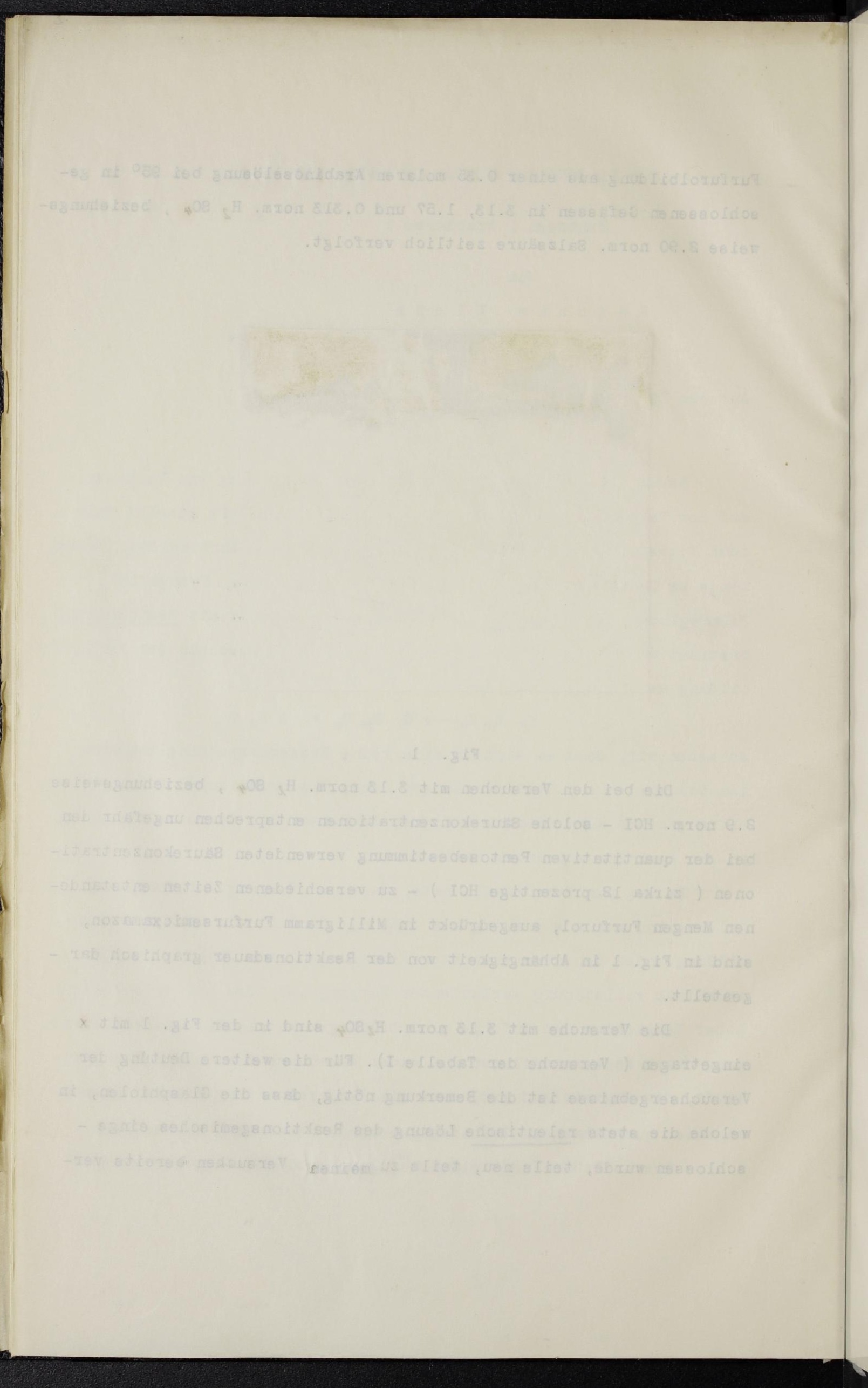


wendet worden waren. Letztere wurden dann jedesmal vor dem neuen Gebrauch mit Chromatschwefelsäure von dem an den Gefässwänden ge bildeten braunschwarzen Belag reingewaschen.

Bei Betrachtung der in Fig. 1. dargestellten Versuchsergebnisse sieht man ein ganz uberraschendes Resultat. Trotz gleicher Versuchsbedingungen ordnen sich die Versuche in zwei streng von einander zu scheidende $d x / d t-K u r v e n ~ A$ und $B$, ohne dass man von vornherein sager kann, aus welchen Grüden bei den einzelnen Versuchen die Funkte tio. in die eine oder die andere Kurve fallen. Es handelt sich also hierbei nicht um allgemein sicher produzierbare Versuche, sondern lediglich um zwei Reaktionsmöglichkeiten, von denen nach Umständen die eine oder die andere eintritt, ohne dass man es in der Hand hat, die eine oder die andere willkürlich zu erzwingen.

Das Bild der Erscheinungen ist ein ganz gleiches, wenn man die Versuche unter sonst gleichen Umständen in 2.9 norm. HCl statt 3.13 norm. $\mathrm{H}_{2} \mathrm{SO}_{4}$ durchfuhrt. Die Versuchsergebnisse fallen entweder in die Kurve A oder B (siehe die Punkteoin Fig.I und die Werte der Tabelle II des experimentellen Teiles).

Da wir für beide Lösungen praktisch annähernd gleiche wasserstoffiorenkonzentration annehmen können, düfen wir schliessen, dass die spezifische Natur der Säure, also ihr Anion, fü den gesammten Erscheinungskomplex ohne Bedeutung ist, sondern dass dieser vielmehr bedingt wird durch die Wasserstoffionen und deren Konzentration. Betrachten wir nun den Typus der beiden Kurven A und B. Nach dem eingangs Gesagten war anzunehmen, dass die Furfurolbildung aus Arabinose in wässeriger Lösung, also einem starken Wasseriberschuss, den Gesetzen einer Reaktion erster Ordnung gehorchen wüde, wobei die Peaktionsgeschwindigkeit proportional der Wasaerstoffionenkonzentration sein wirde.

Die Kurve A zeigt einen fur autckatalytische Vorgänge typischen Verlauf. Ich habe daher die dieser Kurve entsprechenden Versuche an 


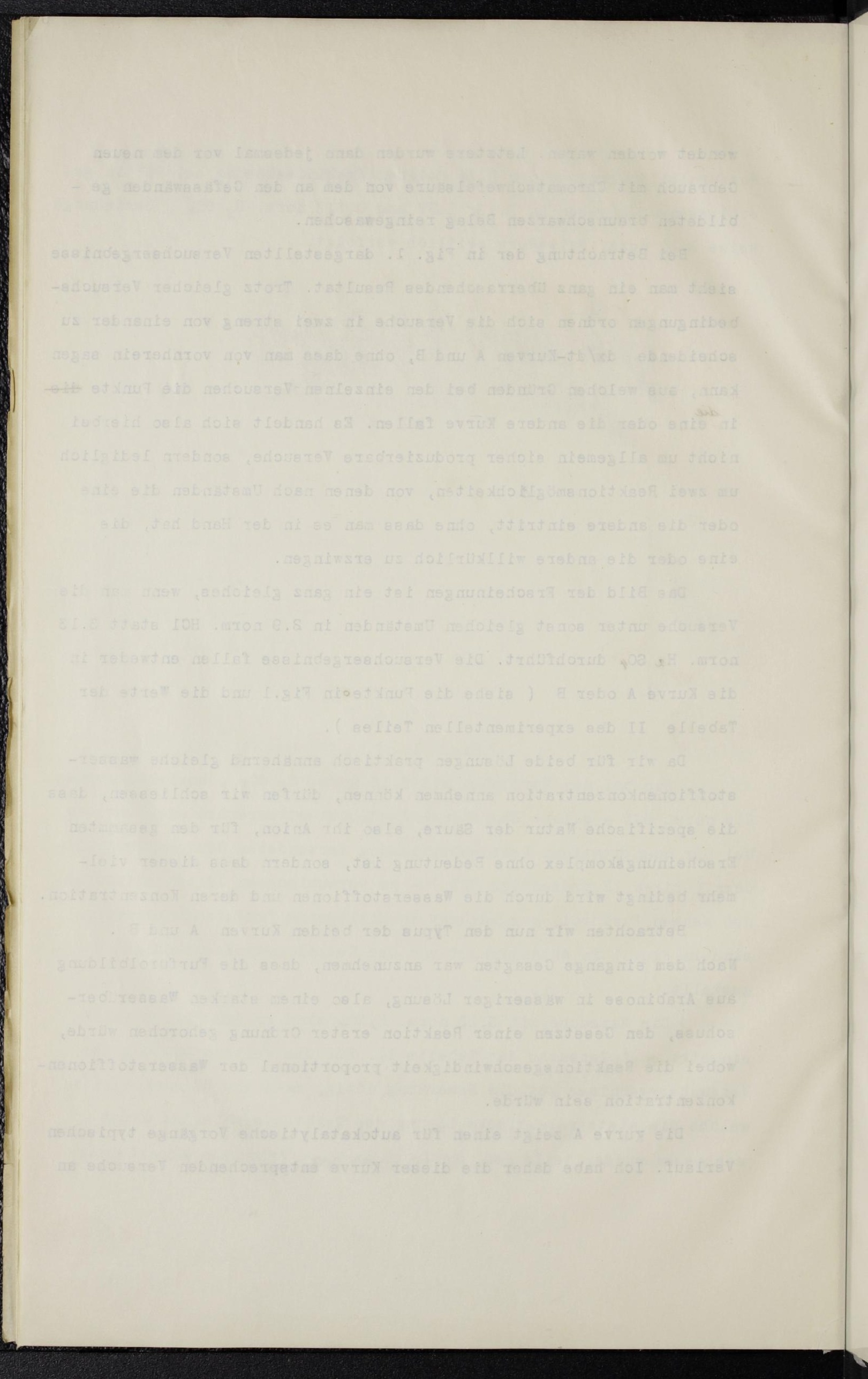


der Formel

$$
d x / d t=\left(k_{1}+k_{2} x\right)(a-x)
$$

gepruft. In integrierter Form lautet diese:

$$
\frac{1}{k_{1}+k_{2} a} \ln \frac{a\left(k_{2} x+k_{1}\right)}{k_{1}\left(\frac{x}{a}-x_{1}\right)}=t .
$$

Setzen wir fur

$$
k_{2}=\frac{k_{1}}{a-2 x_{m}}
$$

und fir

$$
\text { a. }-2 y_{m}=R \text {, }
$$

wo $x_{m}$ die Menge des dem Wendepunkt der Kurve entsprechenden Umsatzes (Niederschlages) bedeutet, wix

$$
k_{1}=-\frac{1}{t} \cdot \frac{R}{2} \frac{a}{a}-2 x_{m} \ln \frac{a}{R}(x-P)(a-x) .
$$

Fur die besprochenen Versuche hat $x_{m}$ den Wert 360 mg ( o Punkt w in Fig. 1).

Wie aus den Tabellen I und II zu sehen ist, lassen sich die Ver suche der Kurve A - bis auf die kleinen Werte von t, die also dem Anfang der Reaktion entsprechenden - ziemlich befriedigend durch die Formel (1) ausdrucken. Es scheint also bezüglich der Vef suche der Kurve A ein ziemlich reiner autokatalytischer Jorgang erster ordnung vorzuliegen .

$$
\begin{aligned}
& \text { Bei Anwendung der Formel erster ordnung auf die Kurve } B \\
& \qquad k=\frac{1}{t}-1 n-\frac{a}{a-x}
\end{aligned}
$$

-wo a wie oben in Formel (1) die Anfangakonzentration der Arabinose bej deutet, die, ausgedruckt in Milligramm des Furfurol-Semioxamazidniederschlages, 955 beträgt - ergab sich, dass die Werte von k im Verlauf der Reaktion ziemlich stark abnehmen. Es kann sich also bezliglich der in die Kurve $B$ fallenden Versuche keinesfalls ur eine Reaktion erster Ordnung handeln. Die Abnahme der Konstanten ist wahrscheinlich auf den Verlaufeiner vebenreaktion zurückzufuhren. Infolge der längeren Reaktionsdauer bei verhältnismässig hoher Temperatur und gleichzeitiger hoher Säurekonzentration düften eben Arabinose oder Furfurol oder beide gleichzeitig eine weitergehende Zersetzung in nicht fassbare Zersetzungs 


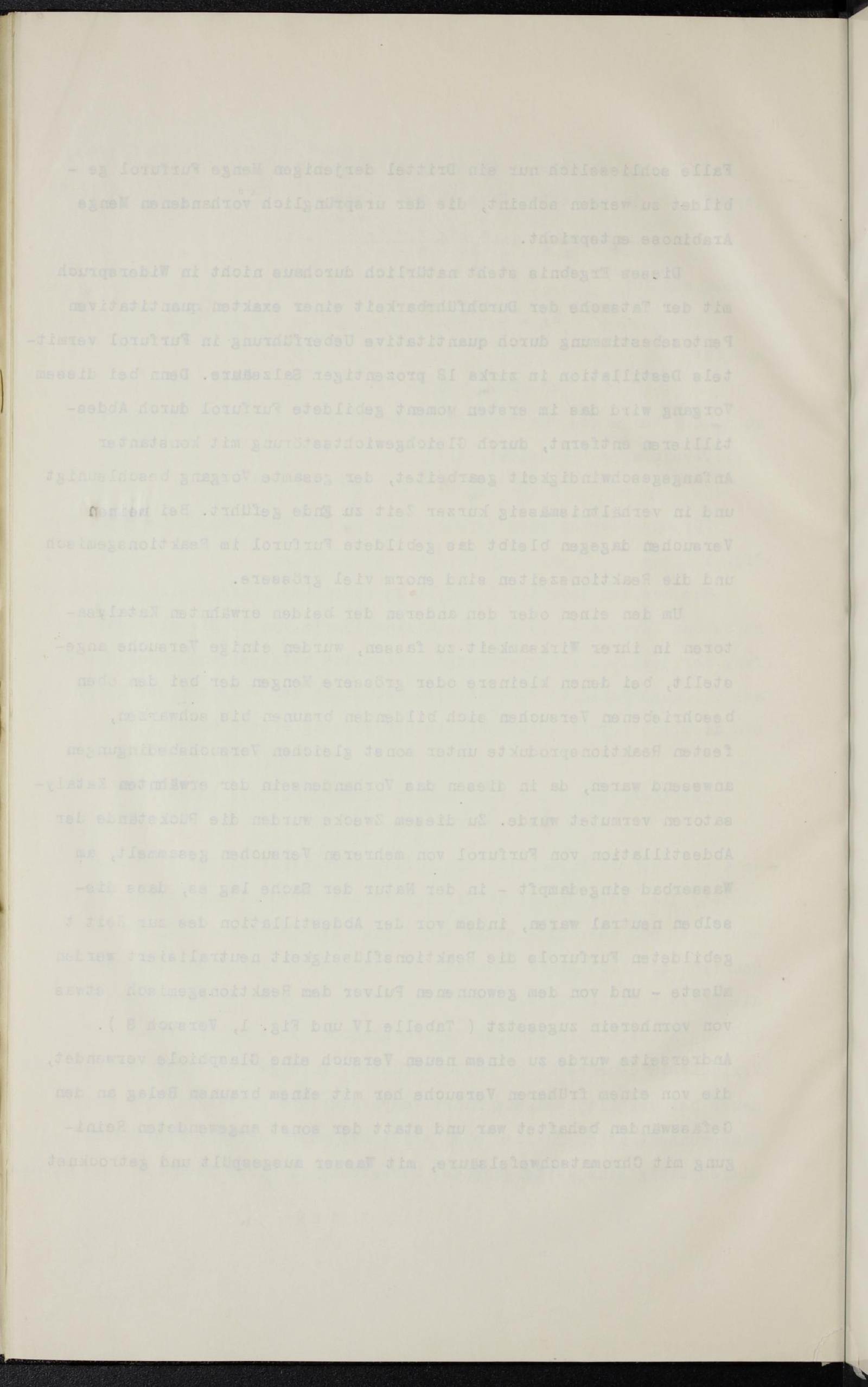


produkte erleiden. Das Hervortreten solcher Nebenreaktionen, also damit das Eintreten des der Kurve B entsprechenden totalen Reaktionsverlaufes, dürfte der Wirkung eines sich während der Reaktion unter Umständen bildenden Katalysators bedingt sein ( Katalysator B).

Was nun den Eintritt des der Kurve A entsprechenden Reaktionsverlaufes anlangt, war die Annahme naheliegend, dass hier, indem der erwähnte Katalysator B hier nicht in Wirksameit tritt ( nicht oder nur in untergeordnetem Masse gebildet wird), das gebildete Funfurol selbst der Beschleuniger ist. Einschlägige Versuche ( Tabelle III), bei denen dem Reaktionsgemisch von vornherein eine be stimmte Furfurolmenge zugesetzt war, ergaben jedoch, dass diese Vermutung nicht zutreffen kann. Denn die bei diesen $\nabla e r s u c h e n ~ z u$ verschiedenen Zeiten neugebildeten Mengen Furfurol sind nicht grösser als den Terten der Kurve A entspricht, sondern geradezu kleiner und entsprechen mehr oder minder den Funkten der Kurve B (Versuche F der Fig. 1).

Es supfoniert also bei diesen Versuchen wieder die Wirksamjeit des Katalysators $B$.

Da sich die der Kurve A entsprechenden Versuchsresultate aber in einiger Annäherung durch die Formel ( 1) ausdrucken lassen, ist anzunehmen, dass sich während der Peaktion proportional der gebildeten Menge Furfurol ein Beschleuniger bildet ( Katalysator A), der den Kurvenverlauf A bedingt.

Zusammenfassend lassen sich also die bisher besprochenen Versuchsergebnisse etwa folgendermassen deuten:

1. Bei $95^{\circ} \mathrm{kann}$ in Arabinoselösungen höherer Wasserstoffionen konzentration sich je nach Umständen, die jedoch nicht regelbar sind, im Verlaufe der Reaktion entweder ein positiver Katalysator A bilden, der die Furfurolbildung also autokatalytisch beschleunigt, oder ein Katalysator B, der Nebenreaktionen beschleunigt, die zur Zersetzung der reagierenden Stoffe fuhren, so dass im letzteren 

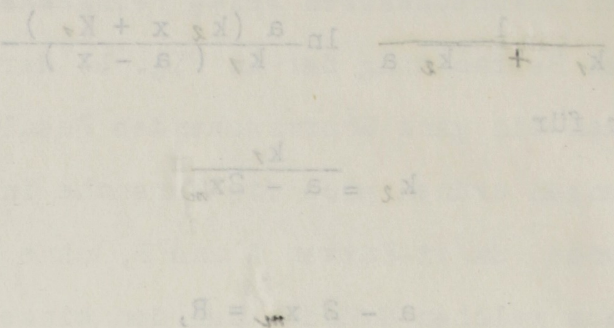
Falle schliesslich nur ein Drittel derjenigen Menge Furfurol ge bildet zu werden scheint, die der ursprünglich vorhandenen Menge Arabinose entspricht.

Dieses Ergebnis steht naturlich durchaus nicht in widerspruch mit der Tatsache der Durchfuhrbarkeit einer exakten quantitativen Pentosebestimmung durch quantitative Ueberfuhrung in Furfurol vermittels Destillation in zirka 12 prozentiger Salzsäure. Denn bei diesern Vorgang wird das im ersten woment gebildete Furfurol durch Abdestiliieren entfernt, durch Gleichgewichtsstürung mit konstanter Anfangsgeschwindigk eit gearbeitet, der gesante Vorgang beschleunigt und in verhältnismässig kurzer zeit zu Bnde gefuhrt. Bei meinon Versuchen dagegen bleibt das gebildete Furfurol im Reaktionsgemisch und die Reaktionszeiten sind enorm viel grössere.

Um den einen oder den anderen der beiden erwähnten Katalysatoren in ihrer Wirksamkeit zu fassen, wurden einige Versuche angestellt, bei denen kleinere oder grössere Mengen der bei den oben beschriebenen Versuchen sich bildenden braunen bis schwarzen, festen Reaktionsprodukte unter sonst gleichen Versuchsbedingungen anwesend waren, da in diesen das Vorhandensein der erwähnten Katalysatoren vermutet wurde. Zu diesem Zwecke wurden die Ruckstände der Abdestillation von Furfurol von mehreren Versuchen gesammelt, am Wasserbad eingedampft - in der Natur der Sache lag es, dass dieselben neutral waren, indem vor der Abdestillation des zur Zeit $t$ gebildeten Furfurols die Reaktionsflissigkeit neutralisiert werden müsste - und von dem gewonnenen Pulver dem Reaktionsgemisoh etwas von vornherein zugesetzt (Tabelle IV und Fig. I, Versuch S).

Andrerseits wurde zu einem neuen Versuch eine Glasphiole verwendet, die von einem früher Versuche her mit einem braunen Belag an den Gefässwänden behaftet war und statt der sonst angewendeten Reinigung mit Chromatschwefelsäure, mit Wasser ausgespult und getrocknet 


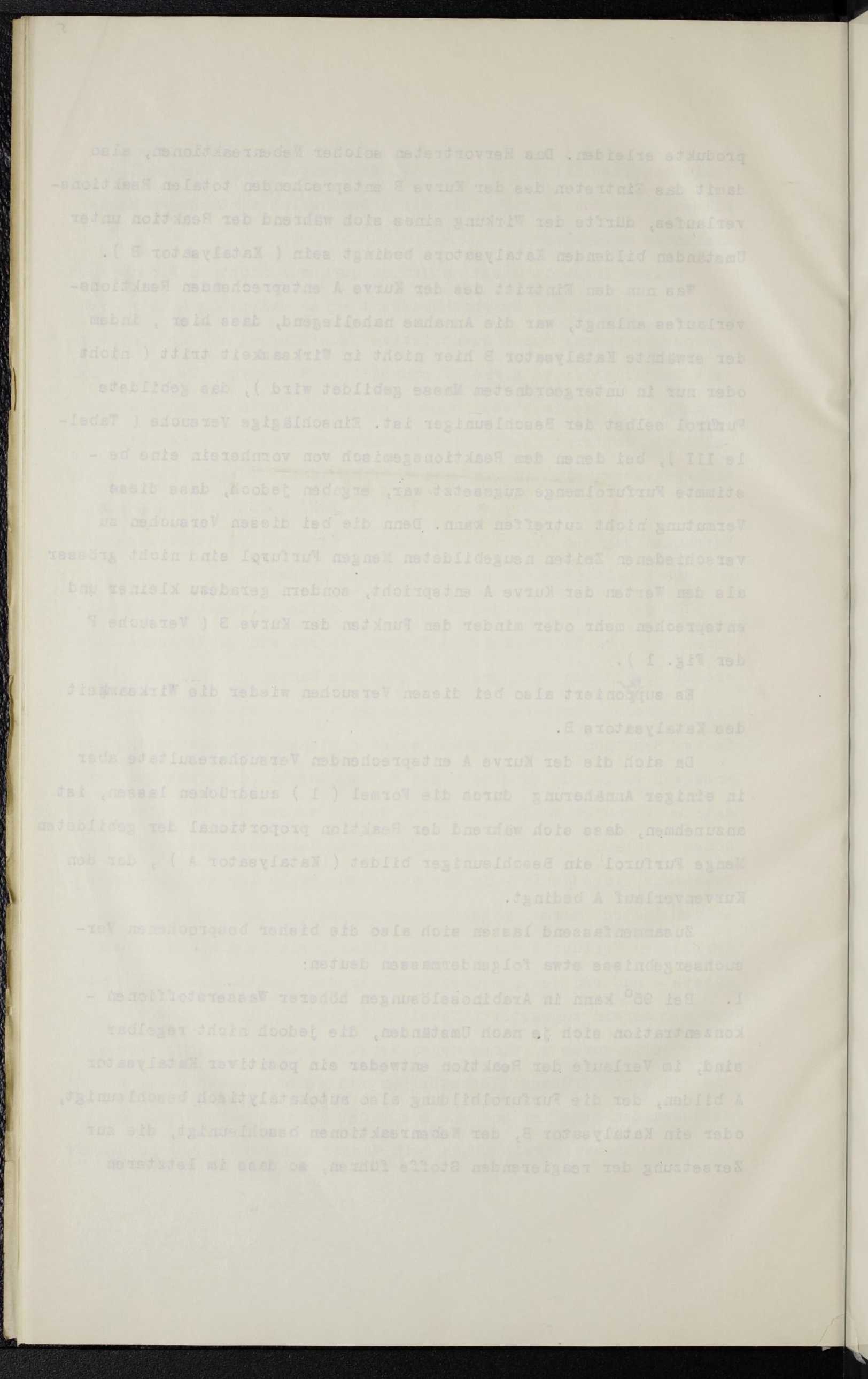


worden war ( Tabelle IV und Fig. 1, Versuch H).

Von allen diesen Versuchen fiel aber keiner, wie aus Fig. 1 zu sehen ist, in die autokatalytische Kurve A, sondern alle nahe in die Kurve $B$.

Es war also auf diese Weise nicht möglich, die Katalysatoren in ihrer Wirksamkeit zu fassen. Möglicherweise sind sie saurer Natur und werden durch die oben erwähnte Neutralisation unwirksarn gemacht.

Die analogen Versuche der Furfurolbildung aus Arabinose in wässeriger Lösung bei $95^{\circ}$ in 1.57 norm., beziehungsweise

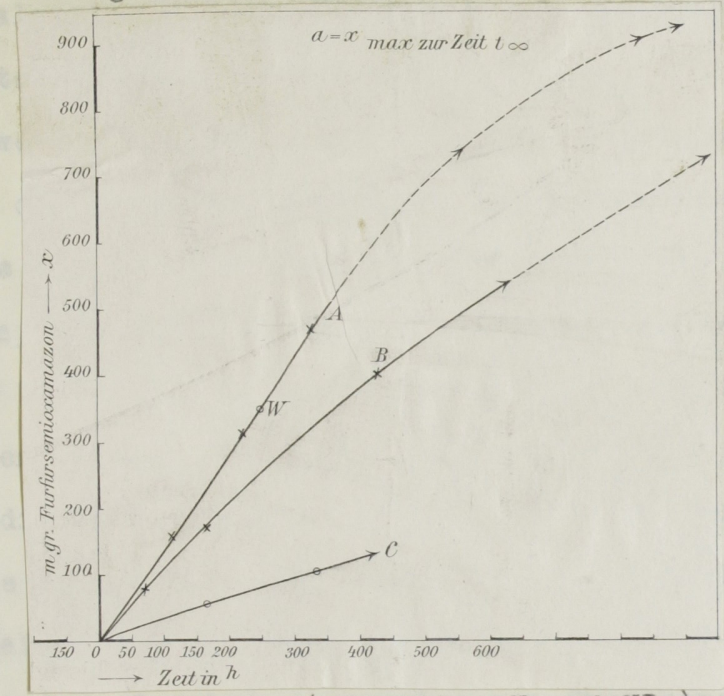

Fig. 2

0.313 norm. Schwefelsäure ( Tabellen $V$ und VI) sind in Fig. 2 graphisch dargestellt. Man sieht, dass auch bei den versuchen in 1.57 norm. $\mathrm{H}_{2} \mathrm{SO}_{4}$ sich die einzelnen Werte von $x$ sich in zwei scharf voneinander getrennte Kurven A und B ordnen lassen, von denen die obere A wieder den Charakter einer autokatalytischen Reaktion zu entsprechen scheint. Doch sind, wie Tabelle V zeigt, die Werte von k, berechnet nach Formel (1), durchaus nicht konstant. Dagegen lassen sich die der Kurve $B$ angehörigen Versuche gut durch die Formel (2) fur eine Reaktion erster Ordnung darstellen.

Während bei den Versuchen mit 3.13 norm. Säure die Kurve B einem Endumsatz zustrebt, der nur etwa einem Drittel der maximal 


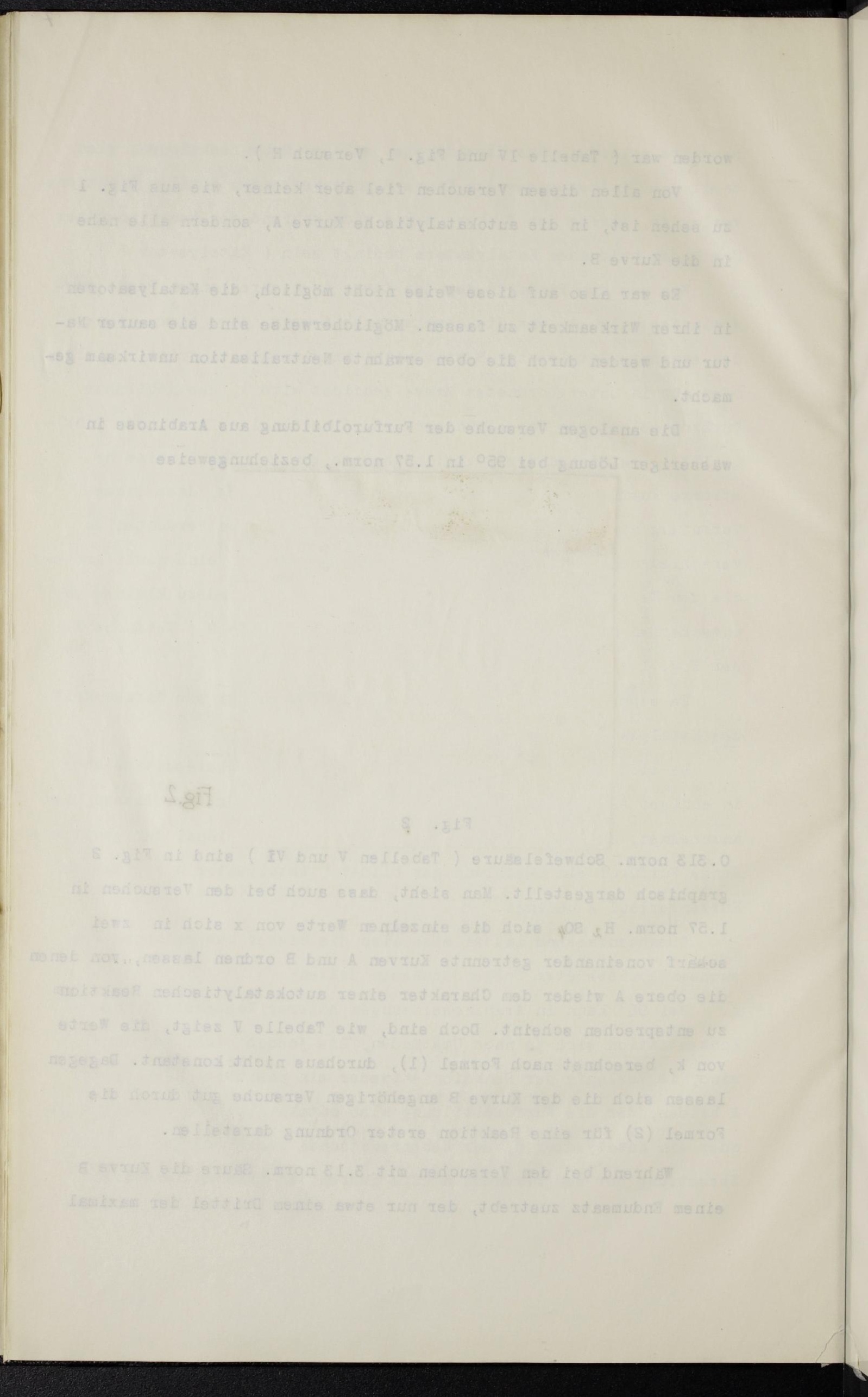


bildbaren Menge Furfurol entspricht (infolge der oben erwähnten, weitgehende Zersetzung bedingenden Nebenreaktionen), deutet der verlauf der Kurve B in 1.57 norm. Säure die Möglichkeit der Er reichung eines Endzustandes an, bei dem praktisch die gesamte mögliche Menge Furfurol gebildet wird, ebenso wie es der Fall ist beim Eintreten des Reaktionsverlaufes nach Kurve A, sowohl in 3.13 norm. als 1.57 norm. Schwef el säure. Das heisst: Herabminderung der Wasserstoffionenkonzentration auf die Hälfte drückt die erwähnten Nebenreaktionen, beziehungsweise die Bildung des dieselben bedingenden Katalysator auf ein Minimum herab, wenigstens innerhalb des von uns untersuchten Umsatzbereiches. 1

Eine weitere Folge dieser Erscheinung ist das Naherrücken der Kurve $B$ an die Kurve A. Es macht mir aber den Eindruck, als ob auch andrerseits die Kurve A herabgedruckt erscheint, d.h. im Sinne obiger Darlegungen, als wenn auch die Bildung des katalytischen Beschleunigers A der Furfurolbildung in schwächer saurer Lösung nicht so stark in den Vordergrund treten wirde.

Auch die Tatsache, dass sich die Werte der Kurve A für 1.57 norm. Säure nicht durch die Formel (1) gut ausdrücken lassen, sprechen dafur, dass die Kurve A keine rein autokatalytisch ist.

Es scheint also bei der geringeren Säurekonzentration auch die Neigung zur Bildung des Katalysators $A$ eine geringere zu sein und auch wenn er gebildet wird, sich der durch ihn beschleunigten Reaktion die einfache. Reaktion erster Ordnung nebenzulagern.

1 Eine zeitlich weitergehende Verfolgung der Reaktion in 1.5 ? norm. $\mathrm{H}_{2} \mathrm{SO}_{4}$ unterblieb wegen der hierzu nötigen allzu langen Reaktionszeiten uber $\underline{500}^{\text {h }}$. 


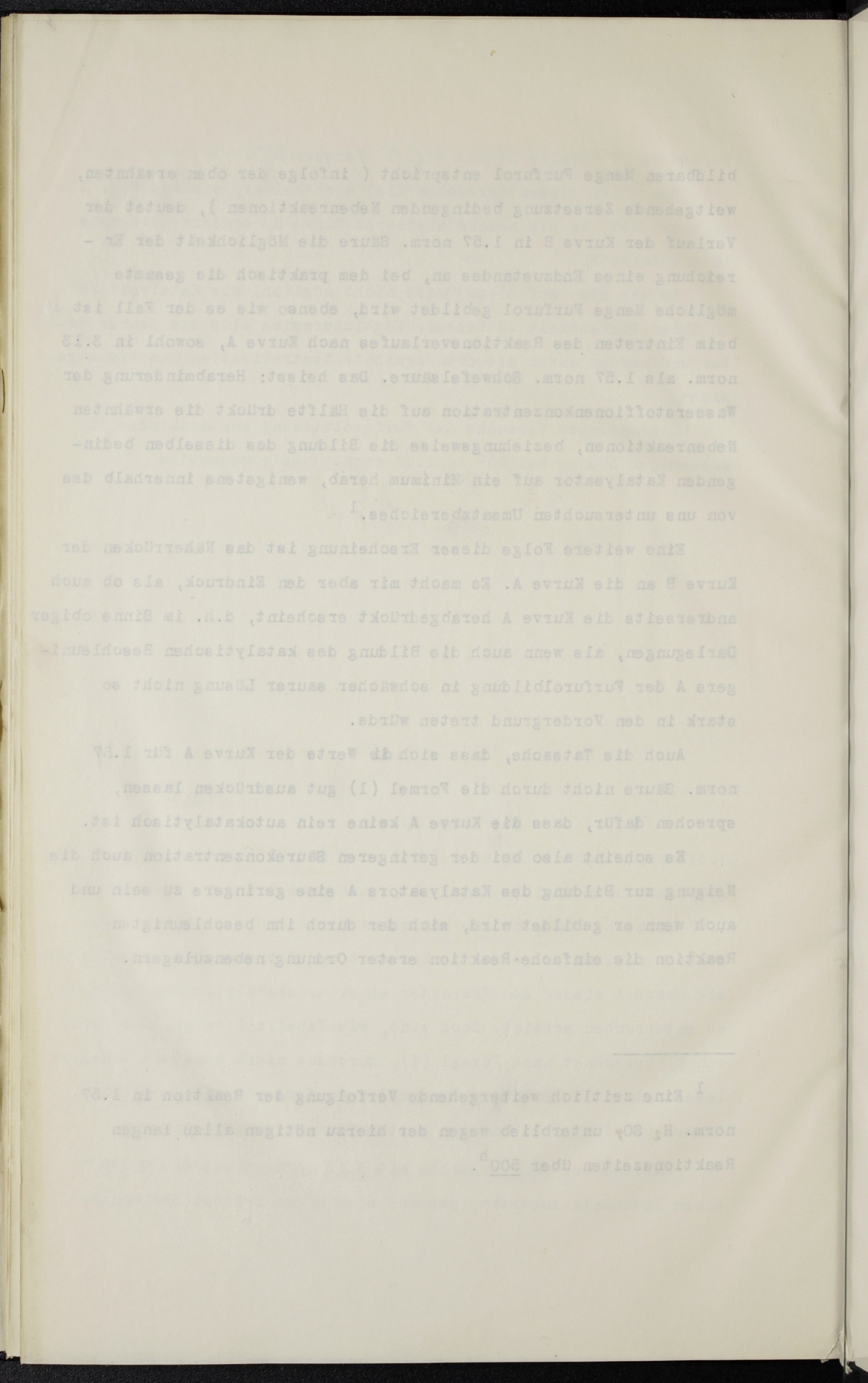


Zusammenfassend darf uber diese Versuche also gesagt werden, dass

2. mit sinkender Säurekonzentration die beiden verschiedenen Kurven A und B immer näher aneinanderzurücken scheinen, um schliesslich in eine einzige, einer Reaktion erster Ordnung entsprechende Kurve zusamen zufallen.

Zwei bei der geringeren Säurekonzentration von 0.313 - norm. angestellte Versuche zeigen denn in der Tat auch gute Konstanz für einen Reaktionsverlauf erster Ordnung ( Tabelle VI). Weitere Versuche in dieser Reihe unterblieben wegen der hierzu nötigen allzu langen Versuchsdauer. Denn mit sinkender Säurekonzentration nimmt die Geschwindigkeit der Furfurolbildung ganz allgemein ab. Ver gleicht man beispielsweise die der Kurve $B$ bei 3.13 norm. und 1.57 norm. Säure und der Kurve c bei 0.313 norm. Säure, beziehungsweise die der Kurven A bei 3.13 norm. und 1.57 norm. Säure gleicher Zeiten, z.B. in 100 Stunden entsprechenden Umsätze $x$

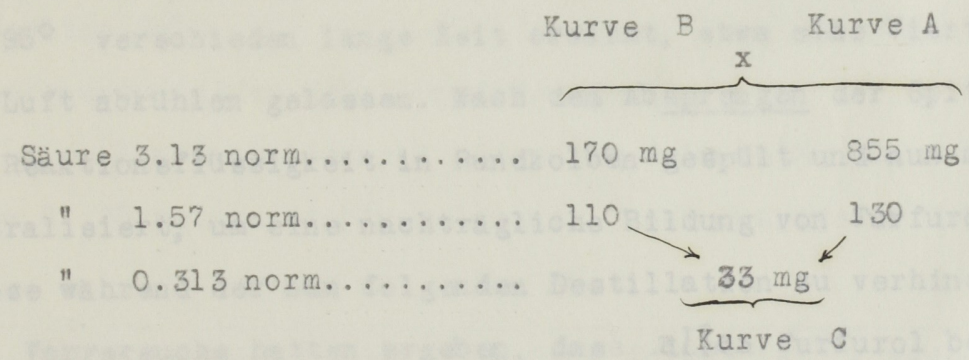

so sieht man deutlich, dass für beide Kurventypen die Herabminderung der Wasserstoffionenkonzentration eine symbth gehende Herabminderung: der Menge gebildeten Furfurols zur Folge hat.

Es dirfte also aller Wahrscheinlichkeit nach die von vornherein vermutete Proportionalität zwischen der Wasserstoffionen konzentration und der Geschwindigkeit der Furfurolbildung vorliegen, die aber durch die oben beschriebenen, gleichfalls von der Wasserstoffionenkonzentration abhängigen Nebenreaktionen, ihrerseits be- 


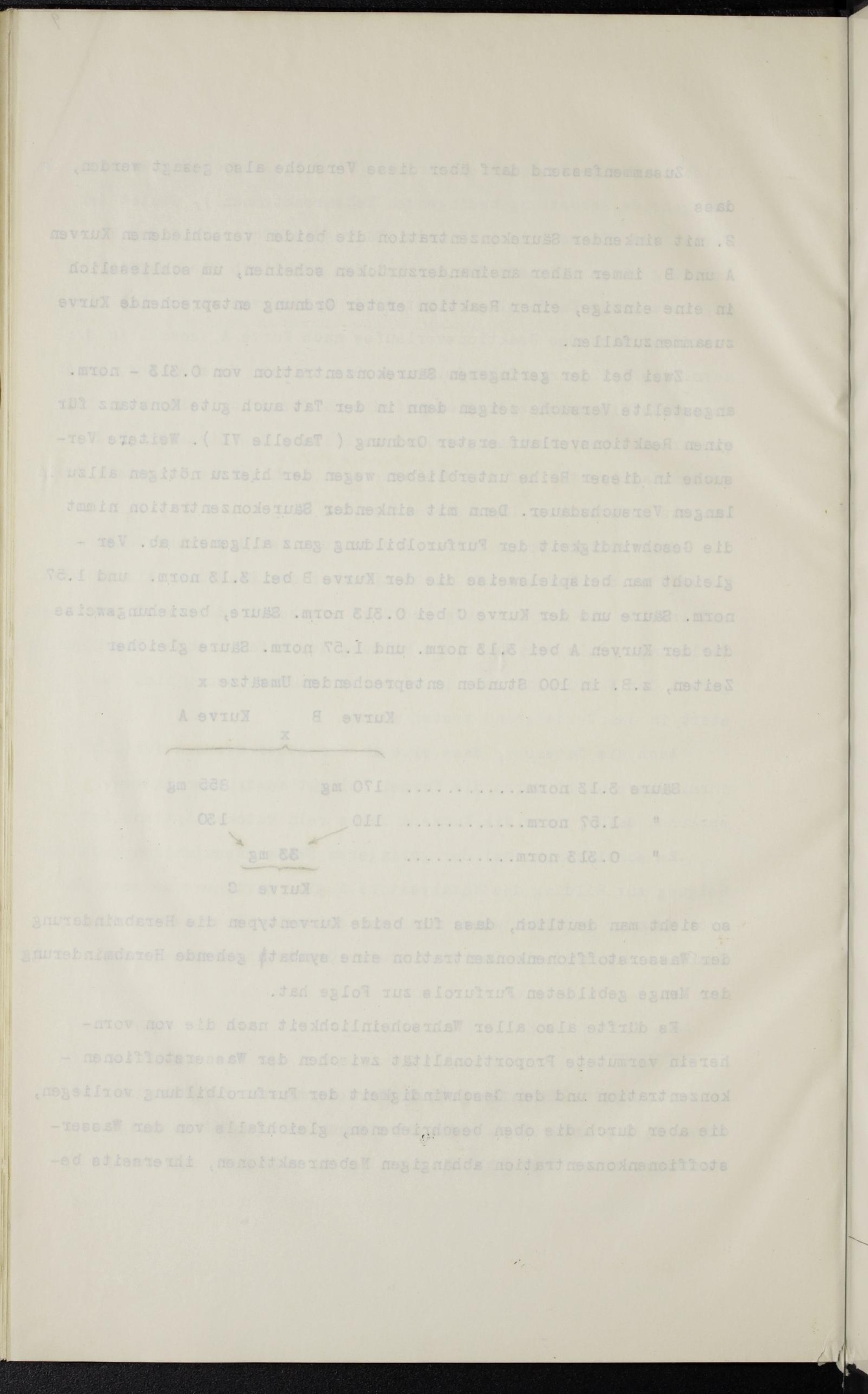


dingt durch die Bildung der Katalysatoren A oder B, mit steigender Konzentration der Wasserstoffionen in steigendem Masse verwischt wird.

Experimente 11 e r $T$ e $i 1$.

Zur Ausfuhrung der beschriebenen Versuche wurden je $5 \mathrm{~cm}^{3}$ einer 1.054 molekularen Arabinoselösung, enthaltend 79 mg Arabinose, in Glasphiolen mit ausgezogener Spitze gebracht und den je, $10 \mathrm{~cm}^{3}$

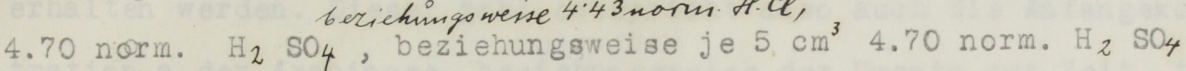
und $5 \mathrm{~cm}^{3}$ Wasser, beziehunggweise je $1 \mathrm{~cm}^{3} 4.70$ norm. $\mathrm{H}_{4} \mathrm{SO}_{4}$ und $9 \mathrm{~cm}^{3}$ Wasser zugegeben und die Glasphiolen zugeschmolzen. Man erhält so Reaktionsflussigkeiten, die in Bezug auf Arabinose 0.35 molekular waren, in Bezug auf Schwefelsäure 3.13, 1.5? beziehungsweise 0.313 normal, in bezug auf Salzsaure 2.9 normal.

Die so vorbereiteten Glasphiolen wurden nun in Thermostaten bei $95^{\circ}$ verschieden lange Zeit erwärmt, etwa eine Viertelstunde an der Luft abkuhlen gelassen. Nach dem Absprengen der Spitze wurde die Reaktionsflussigkeit in Rundkolben gespult und nun mit $\mathrm{NaOH}$ neutralisiert, um eine nachträgliche Bildung von Furfurol aus Arabinose während der nun folgenden Destillation zu verhindern.

Vorversuche hatten ergeben, dass alles Furfurol bei der Destillation übergeht, enn etwa zwei Drittel der Flüssigkeit überdestilliert wurden. Es wurde also stets auf ein so kleines Volumen eingeengt, bis der Rest im Kolben stark zu stossen anfing. Man konnte dann sicher sein, dass alles Furfurol in der Tat Ubergegangen war. In die überdestillierte Furfurollösung und den Spülwässern aus dem Kuhler wurde das Furfurol nun nach $K$ e $p$ und $U n g$ e $r^{l}$ mit Semioxamazid $\mathrm{HH}_{2} \mathrm{CO} . \mathrm{CO} . \mathrm{NH} \mathrm{NH}_{2}$ in ungefähr 0.5 molarer Lösung bei $30^{\circ}$ bis $40^{\circ}$ gefällt. Nach 24 stündigem Stehen (bei stark verdunnten Furfurollösungen nach zwei-bis dreitägigem Stehen) wurde das ge-

\footnotetext{
1 Ber., 30,585, 1897
} 


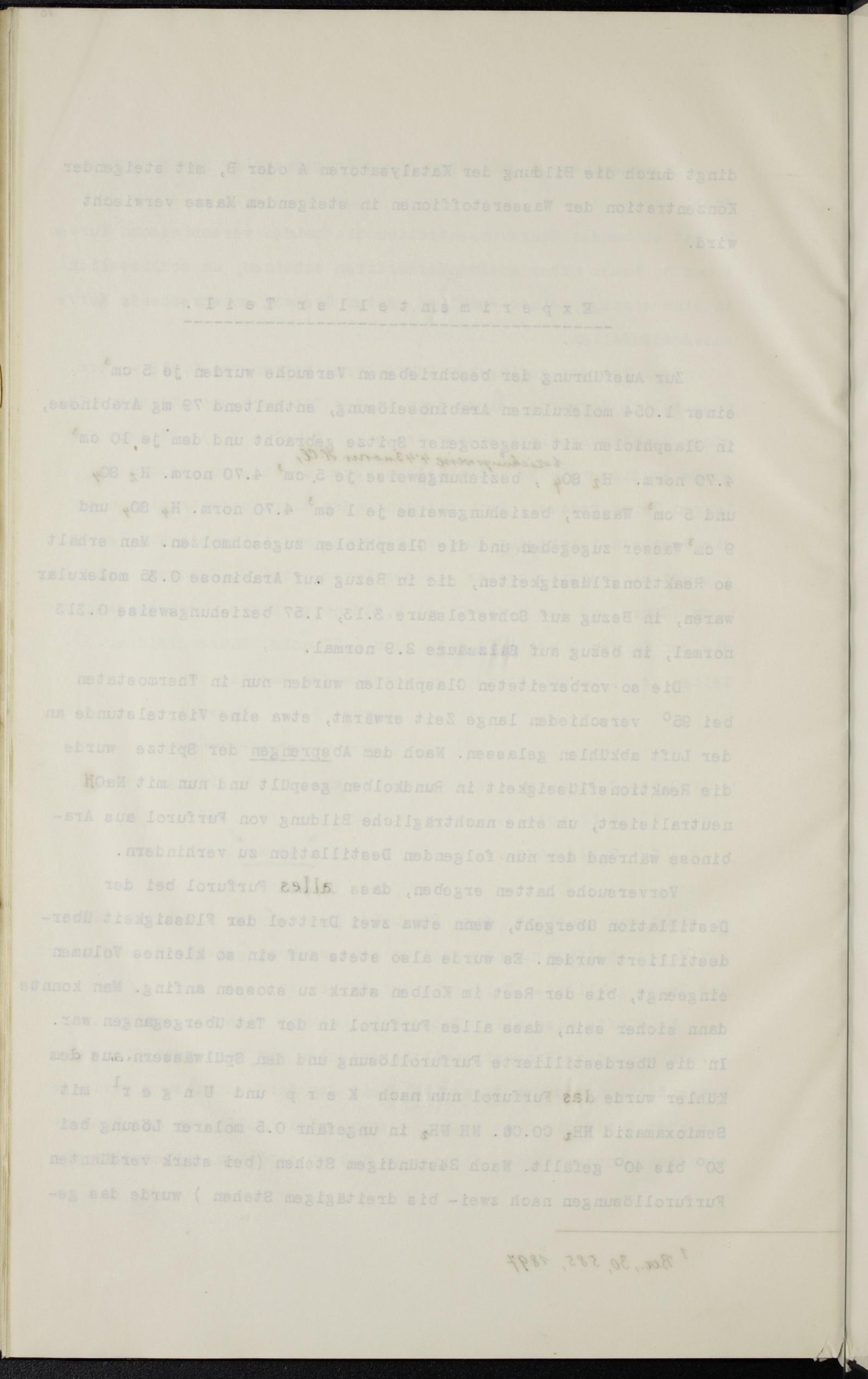


fällte Furfursemioxamazon auf ein gewogenes Filter gebracht, erst mit kaltem Wasser, sodann mit Alkohol und Aether gewaschen. Die alkoholischen und ätherischen Waschflüssigkeiten wurden in einer gewogenen Flatinschale zur Trockene eingedunstet, der Niederschlag mit dem Filter dazu gegeben und nach Trocknung bei $110^{\circ}$ durch 20 Minuten zur Wägung gebracht.

181 Teilen Furfursemioxamazon entsprechen 96 Teilen Furfurol. Da, wie erwähnt, jeder in Verwendung gebrachten Reaktionsflüssigkeit von $15 \mathrm{~cm}^{3}$ ein Gehalt von $79 \mathrm{mg}$ Arabinose entspricht, so würde im Falle vollständigen Umsatzes der Arabinose $955 \mathrm{mg}$ Furfursemioxamazon erhalten werden. Dieser zahl entspricht also auch die Anfangskonzentration a der Arabinose, beziehungsweise der Umsatz zur Zeit to im Falle vollständiger Peaktion.

Ich lasse nunmehr meine Versuchsergebnisse tabellarisch folgen. Die näheren Versuchsbedingungen sind dem Kopf jeder Tabelle ohne weiteres zu en tnehmen.

$$
\text { T a b e } 11 \text { e I. }
$$

Temperatur $95^{\circ}$.

Zusammensetzung der Reaktionsflüssigkeit:

$5 \mathrm{~cm}^{3}$ Arabinoselösung 1.054 molar $+10 \mathrm{~cm}^{3} \mathrm{H}_{2} \mathrm{SO}_{4} 4.70$ normal = $15 \mathrm{~cm} 0.35$ molare Arabinoselösung, beziehungsweise 3.13 norm.

$$
\mathrm{H}_{2} \mathrm{SO}_{4}
$$

\begin{tabular}{|c|c|c|c|}
\hline Zeit in stunden & $\mathrm{x}^{1}$ & $\mathrm{k}$ nach Formel (2) & $\mathrm{k}$ nach Formel (1) \\
\hline 19.5 & 68 & -- & -- \\
\hline 20.5 & 50 & 0.00114 & -- \\
\hline 43.1 & 90 & 0.00100 & -- \\
\hline 45.6 & 76 & 0.00097 & -- \\
\hline 56.8 & 447 & -- & 0.0196 \\
\hline 71.8 & 647 & -- & $0.015 ?$ \\
\hline 93.0 & 160 & 0.00086 & -- \\
\hline 96.8 & 826 & -- & 0.0112 \\
\hline 120.0 & 925 & -- & 0.0151 \\
\hline 146.25 & 223 & 0.00083 & -- \\
\hline 200.25 & 255 & 0.00068 & -- \\
\hline
\end{tabular}

1 Umsatz in Milligramm Furfursemioxamazon. 


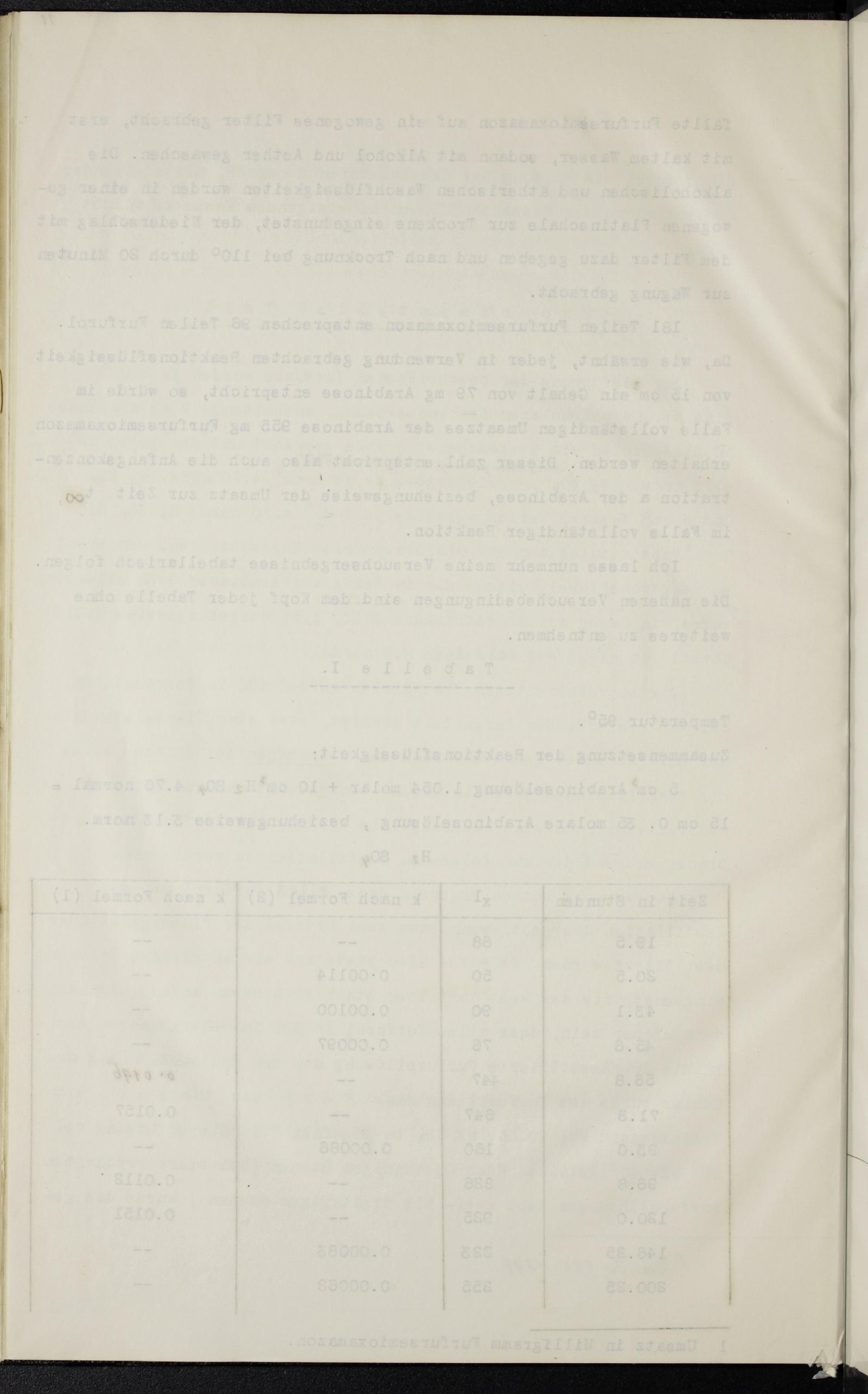


I $a$ b e 1 I e II.

Temperatur $95^{\circ}$. Zusammensetzung der Reaktionsflissigkeit:

$5 \mathrm{~cm}^{3}$ Arabinoselösung 1.054 molar $+10 \mathrm{~cm}^{3}$ HCI 4.43 normal =

$15 \mathrm{~cm}^{3} 0.35$ molarer Arabinoselösung, beziehungsweise 2. Onorm. HI.

\begin{tabular}{|c|cc|c|}
\hline Zeit in Stunden & $\mathrm{x}^{1}$ & $\mathrm{k}$ nach Formel (2) & $\mathrm{k}$ nach Formel (1) \\
\hline 21.1 & 90 & - & - \\
45.8 & 255 & - & 0.0161 \\
103.0 & 172 & 0.00084 & $\ldots$
\end{tabular}

I a $b$ e 1 e III.

Temperatur $95^{\circ}$ Zusammensetzung der Reaktionsflussigkeit:

Lösung von $0.79 \mathrm{~g}$ Arabinose in $5 \mathrm{~cm}^{3}$ einer Furfurollösung vom

Gehalt von $0.313 \mathrm{~g}$ Furfurol $+10 \mathrm{~cm}^{3} \mathrm{H}_{2} \mathrm{SO}_{4} 4.70$ normal.

Die Lösung ist bezüglich Arabinose 0.35 molar, bezüglich Schwefelsäure 3.13 normal und enthält Furfurol, das einer Menge von $f=59 \mathrm{mg}$ Furfursemioxamazon entspricht.

\begin{tabular}{|c|c|c|}
\hline Zeit in Stunden & $\mathrm{x}+\mathrm{f}$ & $\mathrm{x}$ \\
\hline 41.3 & $144 \mathrm{mg}$ & 85 \\
91.3 & 276 & 217
\end{tabular}

T a b e 1 l e IV.

Temperatur 950. Zusammensetzung der Reaktionsflüssigkeit:

$5 \mathrm{~cm}^{3} 1.054$ molarer Arabinoselösung $+10 \mathrm{~cm}^{3} 4.70$ norm. $\mathrm{H}_{2} \mathrm{SO}_{4}$

a) in einer Phiole mit einem Beschlag brauner Reaktionsprodukte (Versuche H);

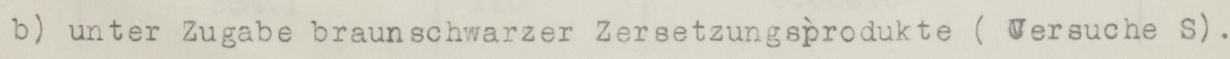

Thusate in mg Furfursemior amazon 


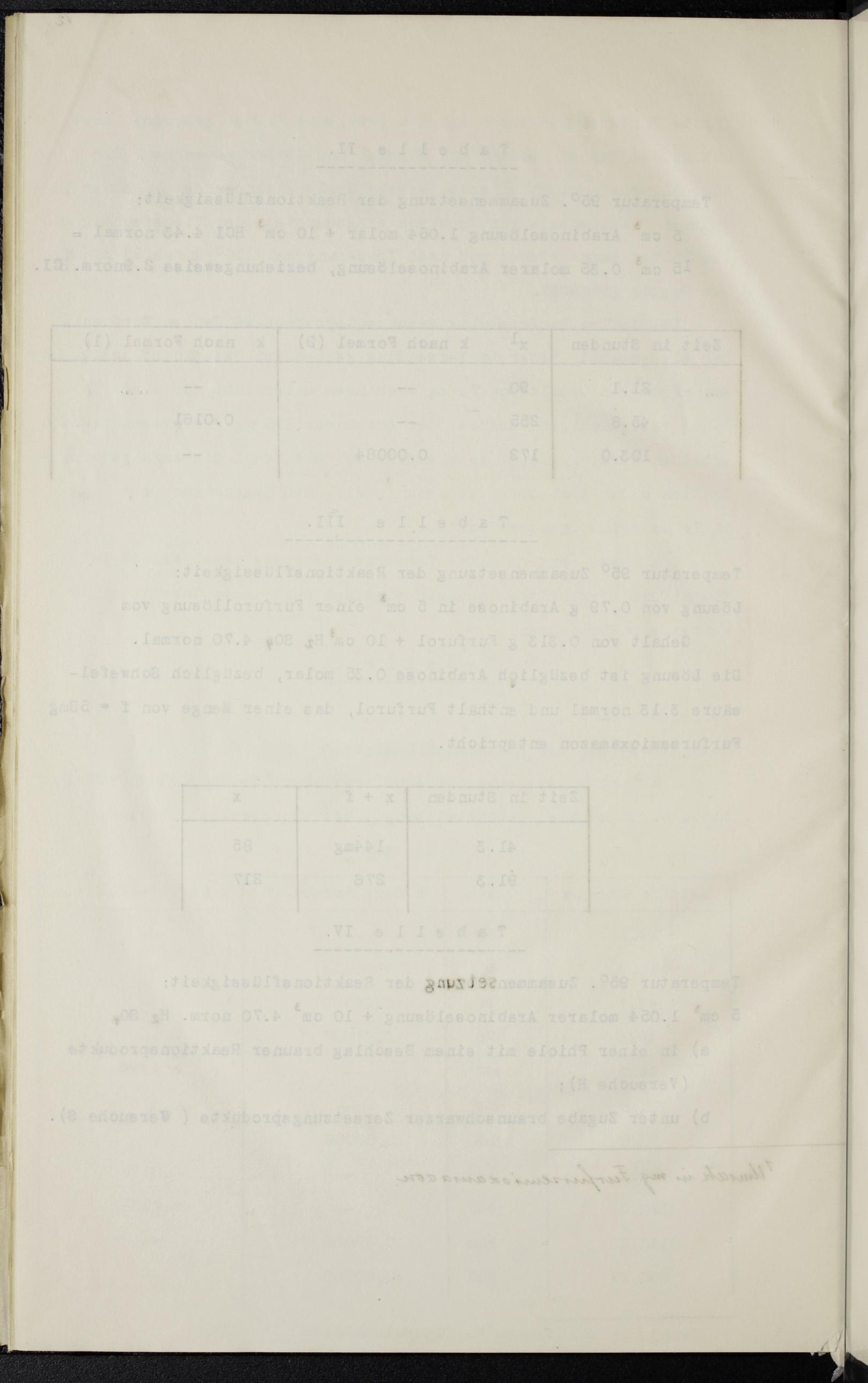




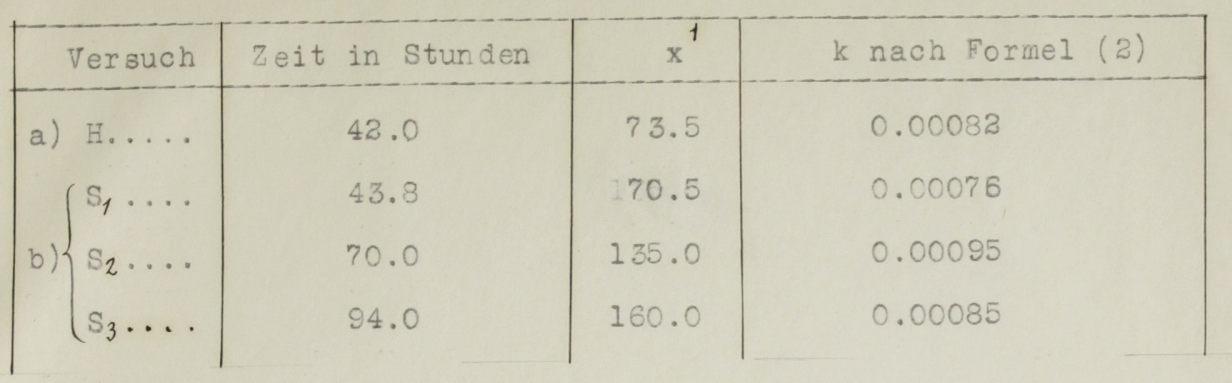

T a b e $\quad \begin{array}{lllll} & 1 & \text { e } & V\end{array}$

Temperatur 950. Zusammensetzung der Reaktionsflüssigkeit:

$5 \mathrm{~cm}^{3}$ Arabinoselösung 1.054 molar $+5 \mathrm{~cm}^{3} \mathrm{H}_{2} \mathrm{SO}_{4} 4.70$ normal +

$5 \mathrm{~cm}^{3}$ Wasser $=15 \mathrm{~cm}^{3} 0.35$ molare Arabinoselösung, beziehungsweise 1.57 norm. $\mathrm{H}_{2} \mathrm{SO}_{4}$

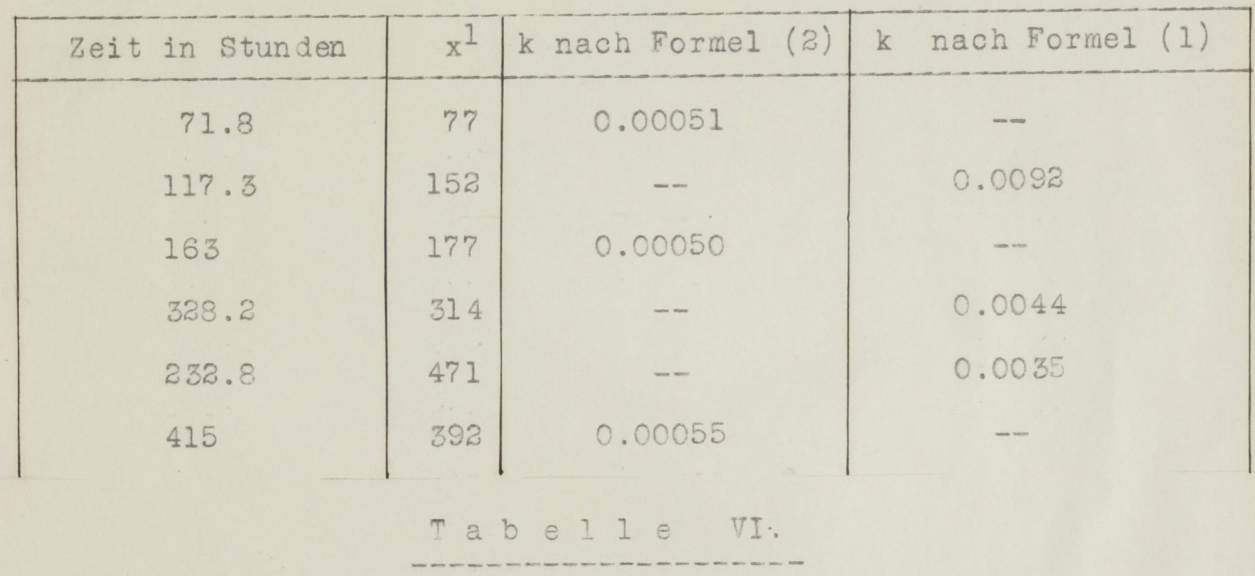

Temperatur $95^{\circ}$. Zusammensetzung der Reaktionsflüsigkeit:

$5 \mathrm{~cm}^{3}$ Arabinoselösung 1.054 molar $+1 \mathrm{~cm}^{3} \mathrm{H}_{2} \mathrm{SO}_{4} 4.70$ normal +

$9 \mathrm{~cm}^{3}$ Wasser - $15 \mathrm{~cm} 0.35$ molare Arabinoselësung, beziehsungsweise 0.313 norm. $\mathrm{H}_{2} \mathrm{SO}_{4}$

\begin{tabular}{|c|c|c|}
\hline Zeit in Stunden & $\mathrm{x}^{1}$ & k nach Formel (2) \\
\hline 167.3 & 55 & 0.00015 \\
334.1 & 110 & 0.00016
\end{tabular}

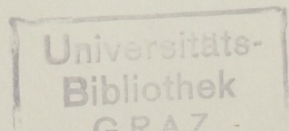

1 Umsatz in Milligramm Furfursemioxamazon. 

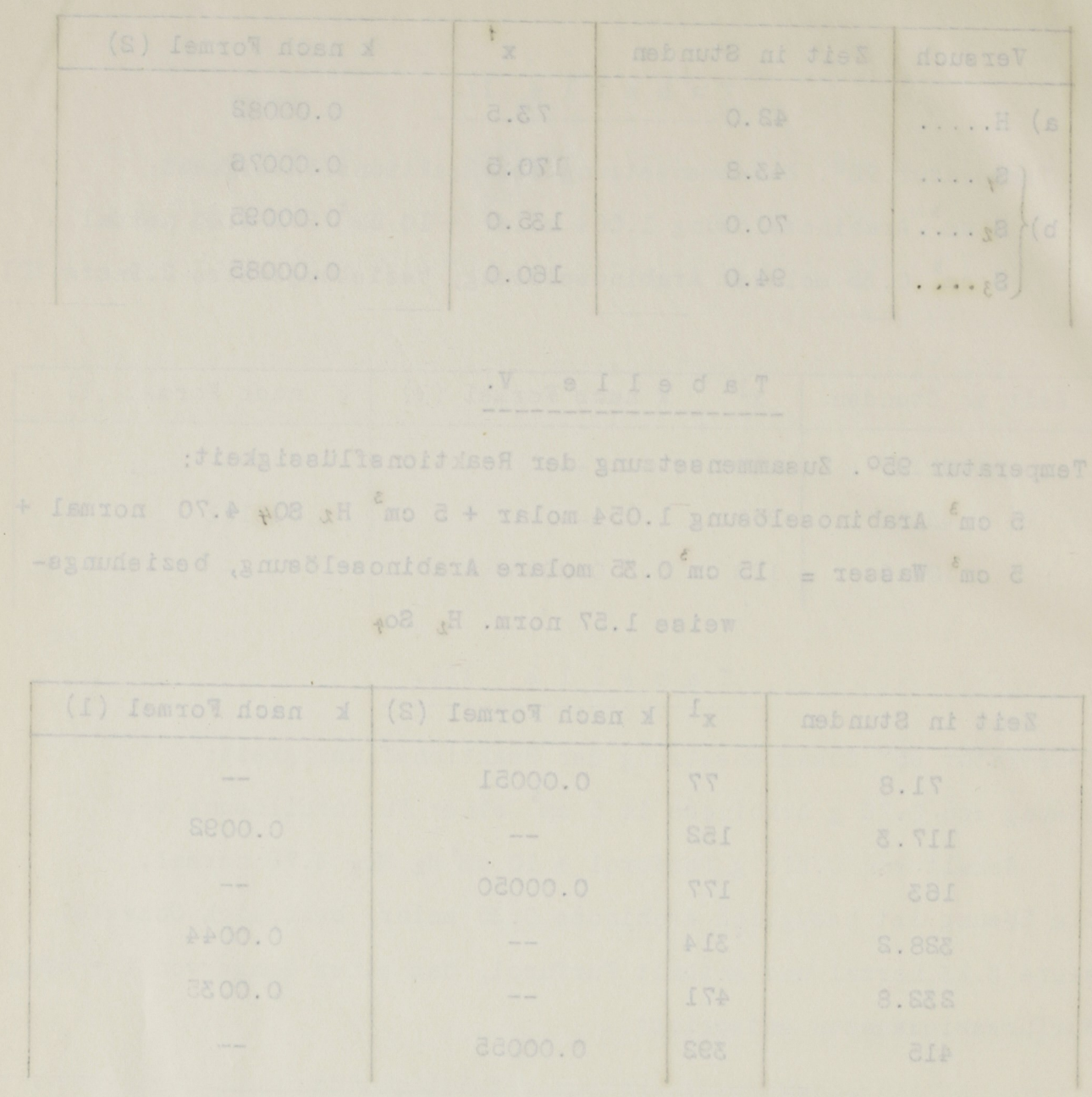



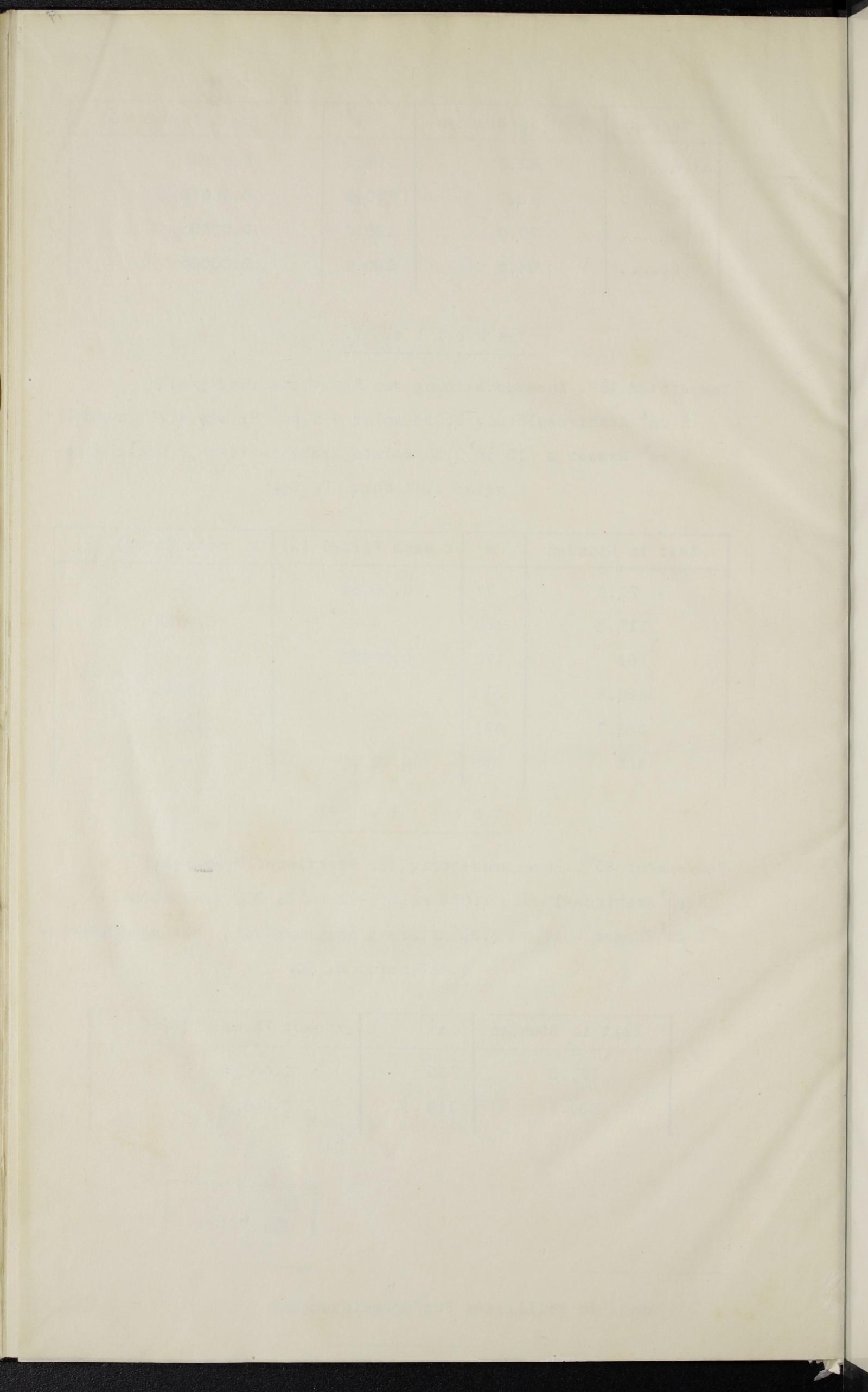





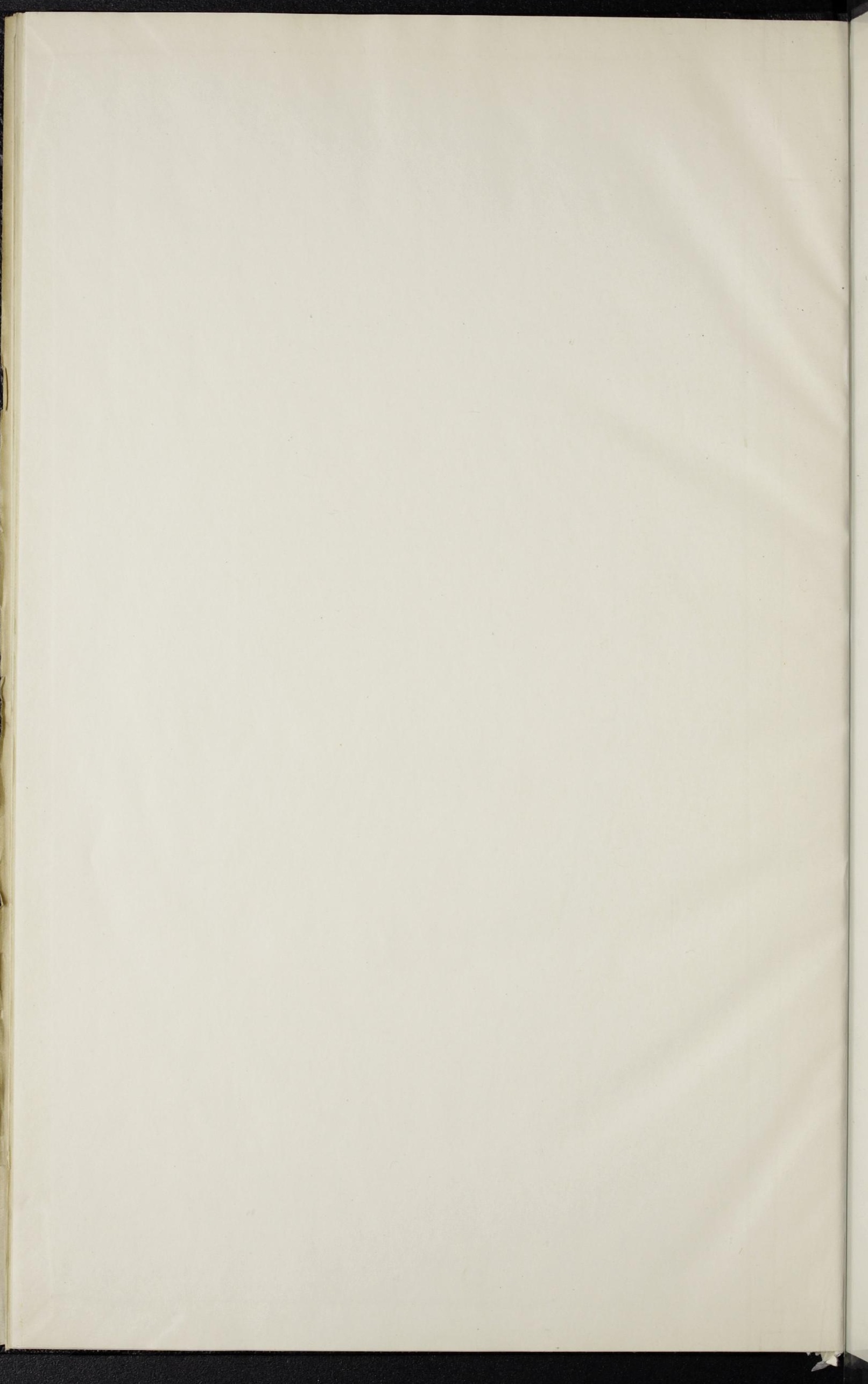



$-(1)$

H. W W

( $610 \%$

H. W W W

ano

Hom in

(2.

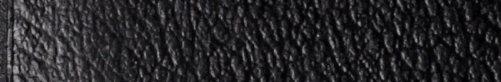

Hown

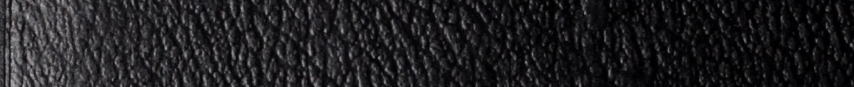

from

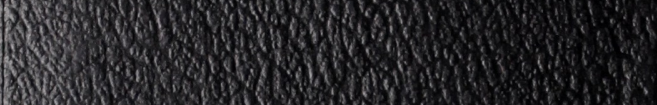

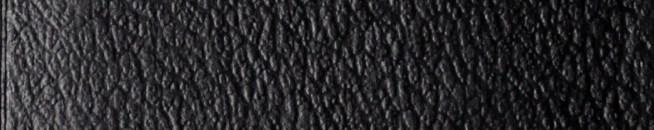

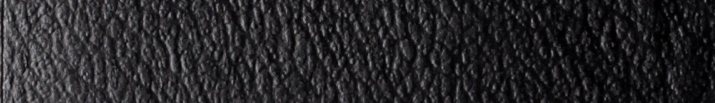

X.

1. . .

H.

12 1 (n)

Wons

W.

3.

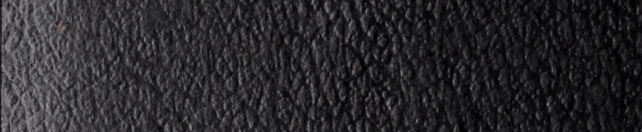

H.

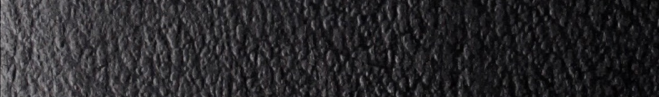

3.

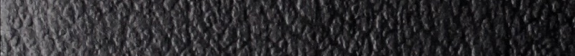

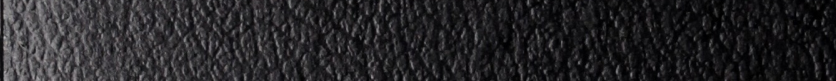

$$
1
$$

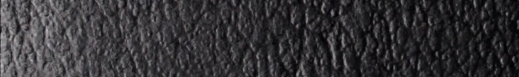

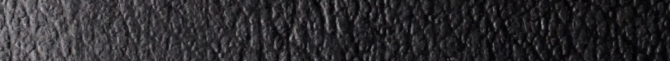

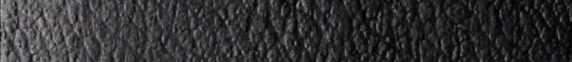

